\title{
Perspective
}

PERSPECTIVE Actualité en histoire de l'art

$1 \mid 2020$

Japon

\section{Transmission du patrimoine architectural au Japon : décryptage}

Un débat entre Nishida Masatsugu, Yagasaki Zentarō et Yoshida Kōichi, mené par Jean-Sébastien Cluzel

Jean-Sébastien Cluzel, Masatsugu Nishida, Zentarō Yagasaki et Kōichi Yoshida

\section{(2) OpenEdition}

Journals

Édition électronique

URL : http://journals.openedition.org/perspective/17541

DOI : 10.4000/perspective. 17541

ISSN : 2269-7721

Éditeur

Institut national d'histoire de l'art

Édition imprimée

Date de publication : 5 juin 2020

Pagination : 43-66

ISBN : 978-2-917902-89-9

ISSN : 1777-7852

Référence électronique

Jean-Sébastien Cluzel, Masatsugu Nishida, Zentarō Yagasaki et Kōichi Yoshida, « Transmission du patrimoine architectural au Japon : décryptage », Perspective [En ligne], 1 | 2020, mis en ligne le 30 décembre 2020, consulté le 24 janvier 2021. URL : http://journals.openedition.org/perspective/17541 ; DOI : https://doi.org/10.4000/perspective. 17541 


\title{
Transmission du patrimoine architectural au Japon : décryptage
}

\author{
Un débat entre Nishida Masatsugu, Yagasaki Zentarō \\ et Yoshida Kōichi, mené par Jean-Sébastien Cluzel
}

Les questions relatives à la transmission du patrimoine architectural ont fait l'objet de nombreux débats dans les années 1990. Les voix de Françoise Choay ${ }^{1}$, Alain Schnapp ${ }^{2}$, André Chastel ${ }^{3}$, Pierre Nora ${ }^{4}$ ont particulièrement marqué ces échanges. Concentrés sur les civilisations européennes, voire méditerranéennes, tous se sont intéressés à la diversité des approches en matière de transmission patrimoniale et, en cherchant un envers aux pratiques occidentales, tous ont été interpelés par la pratique japonaise du shikinen-sengà au sanctuaire d'Ise, rituel dans lequel le sanctuaire est reconstruit à l'identique tous les vingt ans, avant que l'ancien ne soit démonté.

Cette pratique de reconstruction, attestée depuis le VII ${ }^{\mathrm{e}}$ siècle et toujours en vigueur, permettait de remettre en cause, voire de critiquer sévèrement, une certaine hégémonie européenne en matière de conservation architecturale, que les filières patrimoniales et culturelles de l'ONU faisaient alors prévaloir. Dans ces débats engagés, le comparatisme fut une approche privilégiée, qui n'eut pas de pareil pour défaire l'idée d'un fondement universel des pratiques de transmission patrimoniales.

Dans ce contexte, l'Unesco et l'Icomos, dont les travaux visaient la création de chartes internationales sur la conservation des monuments, s'éloignèrent des acteurs en charge de la mise en œuvre des programmes de restauration au sein de différents pays, mais aussi d'universitaires et de conservateurs qui menaient des réflexions historiques et théoriques sur les manières dont les sociétés avaient envisagé de transmettre un héritage tangible ou intangible. Assez rapidement, ces institutions internationales ont essayé de renouer le dialogue entre les différents acteurs du patrimoine, comme en témoigne la Conférence de Nara sur l'authenticité en 19945. Cependant cette conférence n'eut pas l'écho espéré. Les avancées dans la réflexion sur le patrimoine matériel étaient certes importantes, mais elles n'étaient pas suffisantes pour relancer un débat dans lequel chacun puisse se reconnaître. Ainsi les définitions trop restreintes de l'altérité incitèrent de nombreux intellectuels et politiques à se recentrer sur des aires géographiques moins étendues. En France, les six volumes des actes des " Entretiens du patrimoine ", dont les débats furent souvent enflammés, témoignent de ce recentrement culturel sur l'Europe ${ }^{6}$ entre les années 1996 et 2003. Or dans ces débats, certains thèmes, notamment celui 


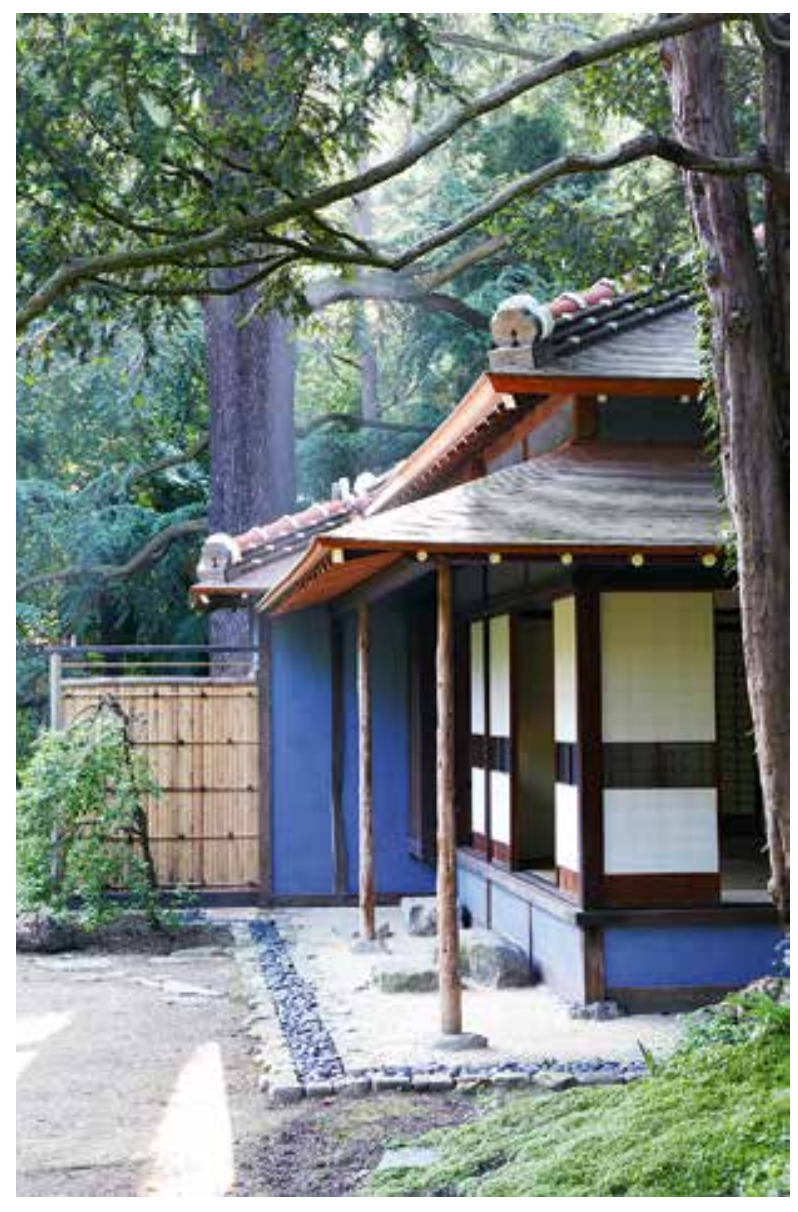

1. Photographie du pavillon Est dans le village japonais, après restauration, jardin Albert-Kahn, Boulogne-Billancourt, 2015-2016. du " patrimoine en place et du patrimoine déplacé " ", abordaient des questions sur lesquelles auraient pu se retrouver l'Asie et l'Europe. Mais les comparaisons de civilisations éloignées étaient, semble-t-il, passées de mode, malgré la parution en l'an 2000 du pamphlet de Marcel Detienne, Comparer l'incomparable ${ }^{8}$, qui défendait les avantages d'un comparatisme constructif entre des civilisations qui a priori n'avaient plus rien à voir les unes avec les autres.

Le débat patrimonial, tant au niveau national qu'international, ne retrouva un certain dynamisme qu'avec l'établissement, par l'Unesco et sous l'égide du Japon, d'une Convention pour la sauvegarde du patrimoine immatériel, en 2003, suivi d'une conférence sur la notion de patrimoine matériel et immatériel ${ }^{9}$ en 2004. Ce thème, plus universel, revigora les échanges scientifiques sur la diversité des systèmes de transmission. Dans ce sens, depuis 2004 en France, d'importantes contributions ${ }^{10}$ sont venues améliorer nos connaissances sur le cadre, les politiques et les systèmes de patrimonialisation japonais. De même, sont aujourd'hui mieux connues en Europe les pratiques de conservation, voire de reconstruction au Japon, celles relatives à l'architecture bouddhique ${ }^{11}$, mais également celles spécifiques à l'architecture shintō, qui relèvent parfois de rituels tel que le shikinen-seng $\bar{u}^{12}$ dont il était question plus avant. Ces avancées dans la connaissance

ont eu d'importantes répercussions, qui vont bien au-delà des domaines théoriques et historiques. Elles ont notamment participé à faire accepter que les pavillons japonais du jardin Albert-Kahn à Boulogne-Billancourt - un patrimoine exceptionnel aux yeux des Japonais et tout à fait unique en Europe - soient restaurés selon des méthodes japonaises traditionnelles, c'est-à-dire qu'ils soient entièrement démontés pour être réparés (fig. 1). Ce chantier ${ }^{13}$, le premier du genre en Europe, en annonce d'autres, mais il est une étape, non pas un aboutissement, dans les échanges franco-japonais autour des questions de la transmission du patrimoine architectural.

Après cette brève introduction qui, d'une certaine manière, résume la vision occidentale sur une question japonaise, on comprendra l'intérêt qu'il y a à donner la parole à des Japonais, comparatistes chevronnés, spécialistes en histoire et théorie de l'architecture, mais aussi impliqués dans de grands projets de restauration, afin de revenir sur la transmission du patrimoine architectural au Japon.

[Jean-Sébastien Cluzel] 
- Jean-Sébastien Cluzel. En matière de conservation du patrimoine, le Japon est souvent perçu comme le contrexemple de l'Occident. En simplifiant, les vieux édifices japonais, les pendants des monuments historiques, auraient, au fil du temps et des restaurations, avec le remplacement des pièces de bois abimées, perdu leur authenticité matérielle. Ces édifices ne conserveraient donc qu'une authenticité formelle. Cette vision, largement partagée en Europe, s'est en grande partie forgée sur l'observation des coutumes de l'archipel, notamment sur le rituel du shikinen-sengū au sanctuaire d'Ise, c'est-à-dire sur la reconstruction cyclique - tous les vingt ans - et à l'identique du sanctuaire depuis le VII ${ }^{\mathrm{e}}$ siècle (fig. 2). Ainsi l'attitude japonaise apparaît-elle inverse de l'occidentale, très ruskinienne, consistant à conserver la matière authentique autant que possible, à réparer en prenant soin de signifier les interventions et, depuis peu, en favorisant les méthodes non intrusives pour diagnostiquer l'état des édifices. Cette vision, dans ses grands traits, correspond-elle à une réalité ou relève-t-elle plutôt d'un imaginaire?

- Nishida Masatsugu. Ce que vous venez de décrire correspond à un résumé de l'un des deux pans de la culture japonaise. Vous avez parlé du shikinen-sengū. Or ce terme, que l'on traduit par «transfert périodique du palais divin », est utilisé en référence à un rituel de renouvellement pratiqué et attesté dans le shintoïsme depuis le VII siècle, rituel au cours duquel les divinités, considérées comme des êtres vivants, se voient

2. Vue aérienne du shōgū (sanctuaire principal) au cœur du sanctuaire intérieur (Naikū) d'Ise, après la reconstruction de 1993. La photographie montre le sanctuaire ancien et le sanctuaire nouveau côte à côte.

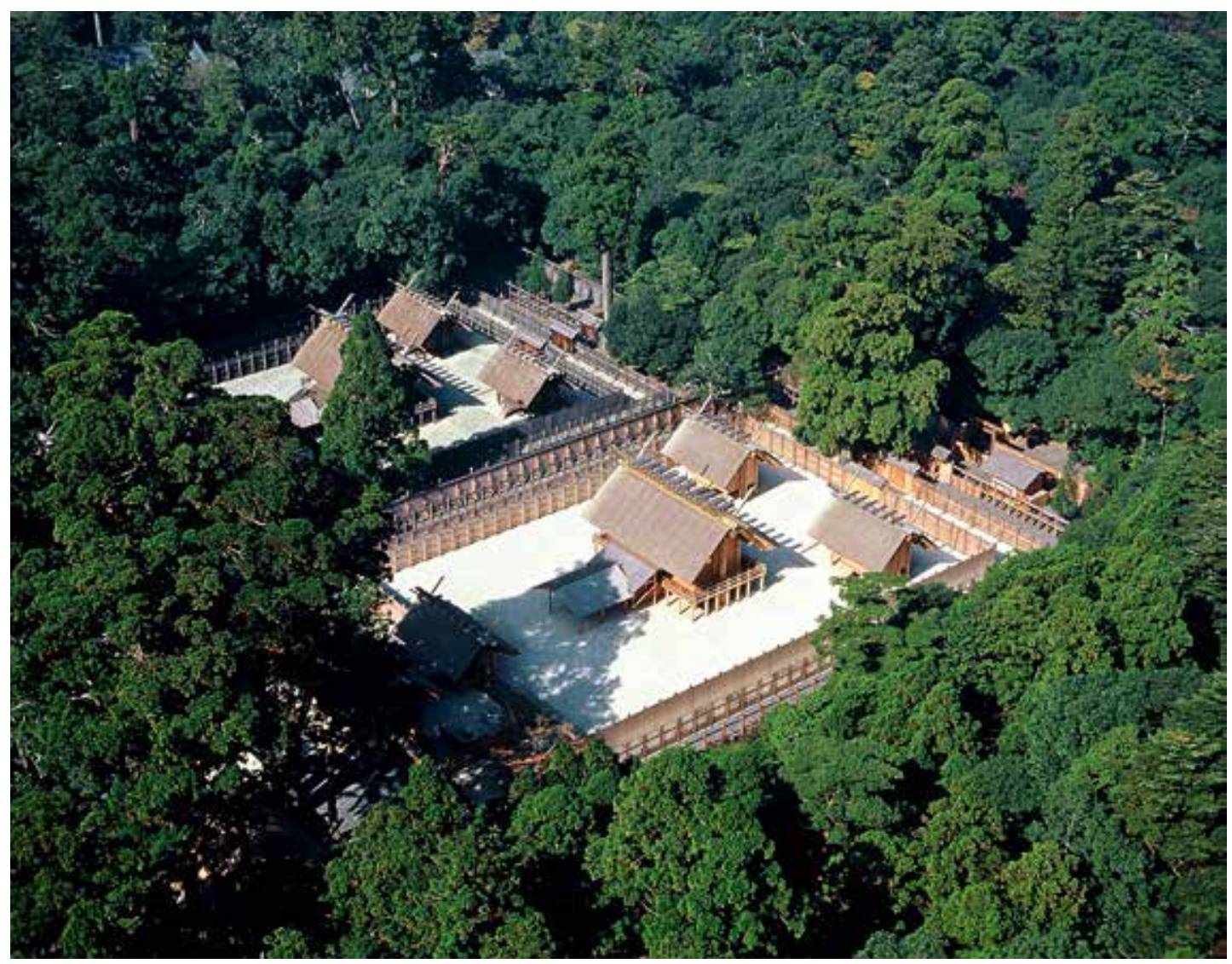




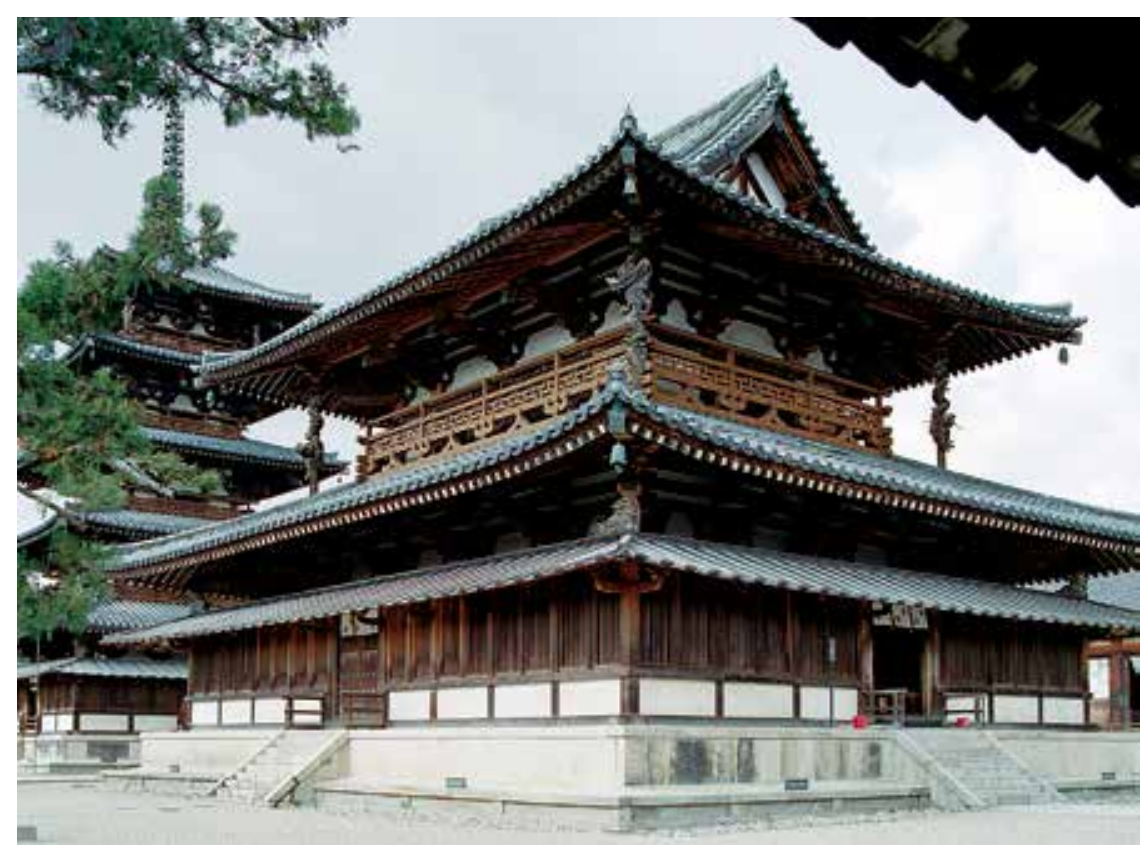

3. Pavillon majeur cultuel (kondō) du monastère bouddhique Hōryū-ji, VII siècle, préfecture de Nara. offrir des repas chaque jour, ont leurs vêtements régulièrement remplacés, leurs chambres entretenues et, le moment venu, leurs demeures renouvelées. Parler du shikinen-sengū c'est donc parler d'un rituel qui englobe, parmi d'autres pratiques, la reconstruction des édifices du sanctuaire. Dans le cadre de ce débat, pour se focaliser sur le remplacement de l'édifice par une reconstruction, il est préférable d'utiliser le terme zōtai et l'expression shikinen-zōtai, qui soustrait la dimension religieuse et permet de se concentrer sur l'aspect constructif de ce phénomène. Mais, je le répète, nous parlons ici d'une pratique liée au shintoïsme et à ses rituels. Or la conservation ou la transmission patrimoniale au Japon ne peut en aucun cas se résumer à un acte religieux. N'oublions pas que la culture japonaise prend également ses racines dans le bouddhisme, autre pan fondamental de notre culture. Et, dans les temples bouddhistes justement, de nombreux édifices en bois, fondés à l'Antiquité, au Moyen Âge ou à l'Époque moderne, ont un degré d'authenticité matérielle équivalent aux grandes cathédrales d'Europe. Je pense par exemple, pour les plus anciens, au pavillon cultuel et à la pagode du Hōryū-ji (fig. 3) qui datent du VII ${ }^{e}$ siècle, ou encore à la pagode du Hokki-ji (fig. 4), de la même époque. La liste des édifices authentiques dans leur matérialité est relativement longue, sans doute plus longue que celle des édifices reconstruits cycliquement avec des matériaux neufs. Enfin, pour ces édifices bouddhiques, il n'est pas question de relier les processus de conservation à des rituels.

- Yoshida Kōichi. Cette question sur la compréhension et l'interprétation des pratiques japonaises depuis l'étranger est intéressante. J'aimerais rappeler que Knut Einar Larsen, spécialiste norvégien de l'architecture en bois, initiateur de la Conférence de Nara sur I'authenticité ${ }^{14}$ (1994), qui connaît très bien la réalité des pratiques de la conservation architecturale au Japon, avait souligné, il a quelques années déjà, que les Européens qui considèrent la méthode japonaise de transmission du patrimoine comme singulière, I'associaient généralement au shikinen-zōtai. Mais cela va sans dire, le shikinen-zōtai ne peut pas expliquer la transmission 
du patrimoine architectural au Japon dans sa globalité. Le professeur Nishida vient de le dire, I'architecture bouddhique n'est pas sujette à des pratiques de reconstruction de type zōtai. En revanche, son ancienneté est appréciée et, s'agissant des temples bouddhiques, on a toujours préféré les réparations aux reconstructions.

Quant à l'origine de la signification du shikinen-zōtai, les avis des spécialistes diffèrent. Pour certains, le zōtai aurait été un moyen de pérenniser des bâtiments provisoires construits à l'occasion d'un rituel, qui consiste à célébrer le retour des divinités parmi les hommes. Ainsi, puisque les bâtiments ont été conçus pour servir de palais provisoires aux divinités, il est tout à fait logique qu'ils aient été périodiquement reconstruits. II n'y a rien de singulier en cela. Pour d'autres spécialistes, l'essence du zōtai relèverait de la transmission des trésors, autrement dit du retour dans le palais divin, c'est-à-dire dans les pavillons cultuels, des régalia qui légitiment la filiation entre les hommes et les divinités. Selon cette seconde interprétation, on retrouve, à l'arrière-plan du shikinen-sengū, l'idée d'un bâtiment provisoire,

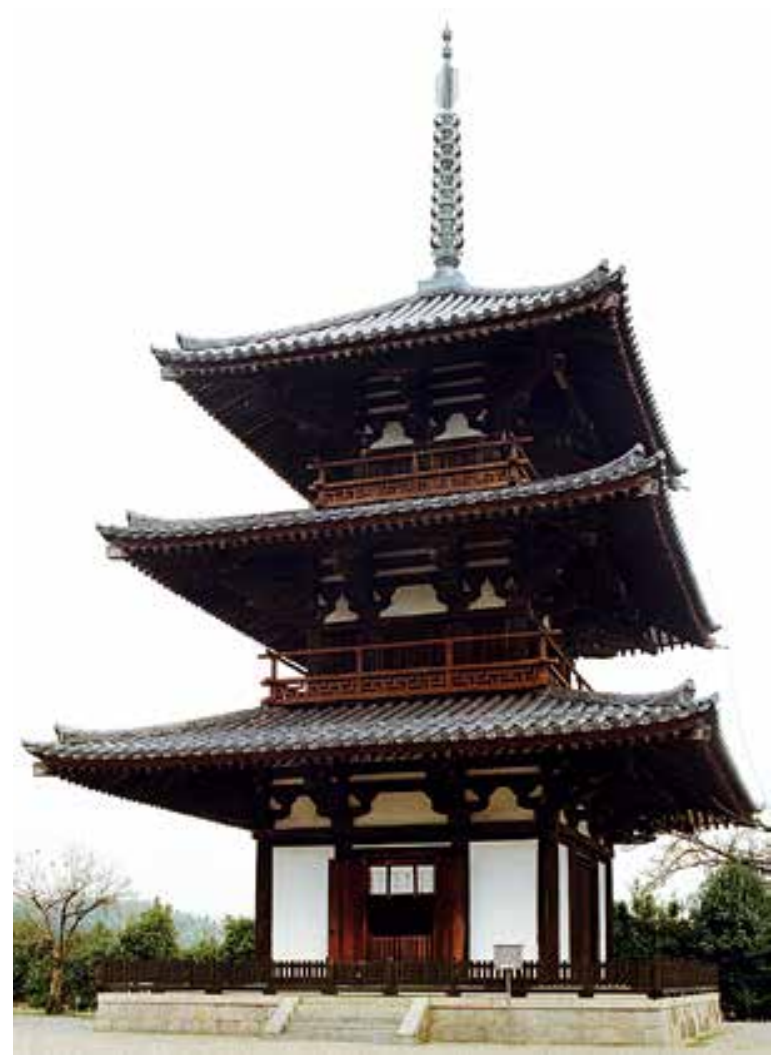

4. Pagode à trois étages du monastère bouddhique Hokki-ji, VII siècle, préfecture de Nara. illustrant le mythe de l'éternel retour, ainsi qu'un goût profond pour les choses neuves. Souvenons-nous que dans l'Antiquité japonaise, la capitale et le palais sont entièrement et systématiquement reconstruits lors d'un changement d'empereur. Mais encore une fois, n'oublions pas que les Japonais sont également extrêmement sensibles à l'ancienneté des choses, sensibilité qui s'exprime par exemple dans une notion déjà attestée au Moyen Âge et, me semble-t-il, très connue en Europe : le wabi ${ }^{15}$.

- Jean-Sébastien Cluzel. Vous êtes tous les deux d'accord sur la présence d'une certaine confusion ou plutôt d'une essentialisation de la pratique du shikinen-zōtai dans la vision occidentale, effaçant par là même les autres pratiques nipponnes de transmission architecturale. Vous rappelez également qu'il n'existe pas de zōtai, c'est-à-dire un certain systématisme dans les reconstructions à l'identique, pour l'architecture bouddhique. Toutefois, cette essentialisation du zōtai est, me semble-t-il, inévitable pour les Européens. Prenons par exemple le Kinkaku-ji, le célèbre Pavillon d'or de Kyōto. Cet édifice a été reconstruit cinq ans après l'incendie criminel qui l'avait dévasté en 1950. De très nombreux Français connaissent ce cas par le biais de la traduction du roman de Mishima Yukio, intitulé Le Pavillon d'or (1956). De plus, cet édifice de la fin du XIVe siècle, qui siégeait au sein de la résidence du shōgun Yoshimitsu, était, au moment de l'incendie, la propriété du temple bouddhique légataire de cette résidence. Comment faire pour éviter cette essentialisation du zōtai dans l'esprit européen, alors que des édifices bouddhiques sont parfois reconstruits à I'identique, et à partir de rien ? 
- Nishida Masatsugu. Dans I'histoire japonaise, il existe trois pratiques traditionnelles pour transmettre le patrimoine architectural. La première, nous l'avons vu, est le shikinen-zōtai. Cette pratique, qui n'a pas de pendant en Occident, permet de transmettre la forme architecturale par des reconstructions cycliques et périodiques. La deuxième pratique vise à faire perdurer l'édifice en effectuant un démantèlement complet ou partiel de celui-ci et en le remontant une fois que toutes les pièces de bois qui le composaient sont réparées. Cette pratique, autrefois appelée zuihashūri, apparaît elle aussi singulière en comparaison des pratiques occidentales. Enfin, la troisième pratique, dans laquelle s'inscrit le Pavillon d'or de Kyōto, est la restitution. Cette pratique permet de ressusciter un bâtiment disparu. Si cette dernière existe aussi en Europe, elle est mise en œuvre beaucoup plus fréquemment au Japon. Ces trois pratiques, distinctes les unes des autres, s'inscrivent dans les traditions japonaises. Toutefois, l'évolution qui s'est opérée à l'ère Meiji (1868-1912) en matière de protection du patrimoine bâti a complexifié leur identification.

- Yagasaki Zentarō. J'aimerais faire une remarque sur la notoriété du zōtai. Si, pour l'Occident, le shikinen-zōtai semble être la pratique de transmission japonaise prépondérante, voire même l'unique, c'est aussi, me semble-t-il, une question de temporalité. Au sanctuaire d'Ise, le shikinken-sengū, le transfert du palais divin, est reconduit cycliquement tous les vingt ans depuis le VII siècle. En 2013 eut lieu la soixante-deuxième reconstruction. Or la probabilité qu'une même personne, japonaise ou non, entende parler au cours de sa vie de ce rituel très particulier est démultipliée par la régularité d'un cycle court de seulement vingt ans. Ce rituel, frappant par son ampleur - quelques cent cinquante édifices sont reconstruits à l'image de leurs pères vieux de vingt ans -, alimente donc sa propre notoriété selon une formule temporelle. En revanche, un édifice

5a. Photographie montrant au premier plan la façade arrière du honden du grand sanctuaire Izumo-taisha dans son état actuel.

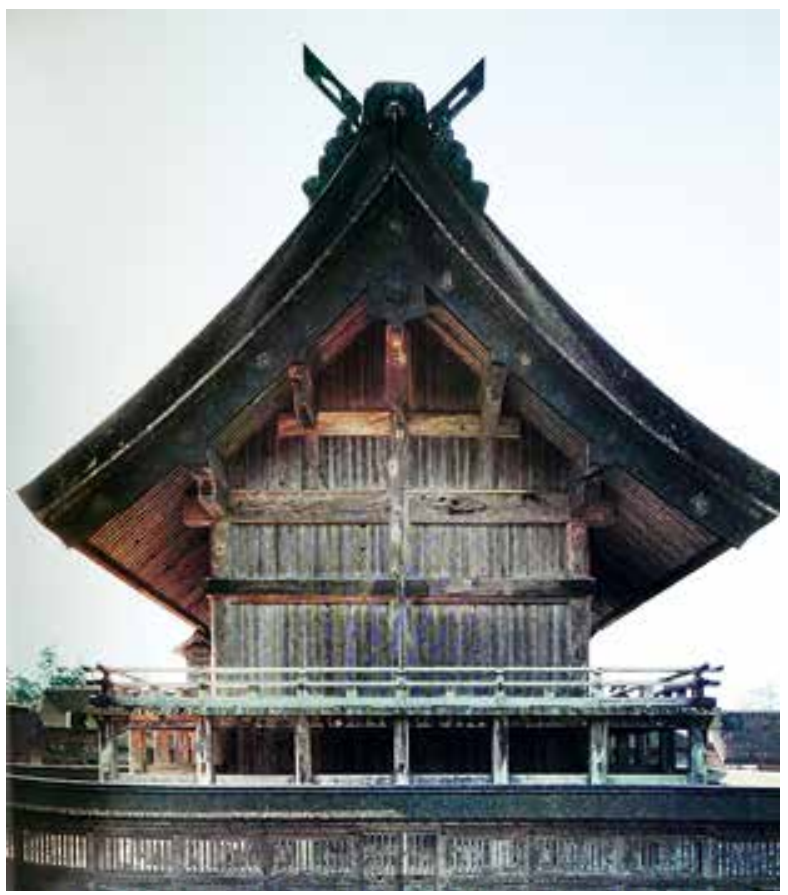
bouddhique, construit dans l'Antiquité ou au Moyen Âge, n'est démantelé pour être restauré en moyenne qu'une fois tous les cent ans, voire une fois tous les trois cents ans pour les plus robustes, c'est-à-dire selon une périodicité très différente. La rareté de ces restaurations participe de leur oubli. Ainsi, aucune campagne de restauration ne peut rivaliser, en termes de notoriété, avec la pratique du shikinen-zōtai qui, depuis l'ère Meiji, est singulière au sanctuaire $d^{\prime} I s e$.

- Jean-Sébastien Cluzel. L'entrée dans l'ère Meiji est un moment charnière dans I'histoire du Japon. Elle correspond à la reprise du pouvoir par l'empereur, à la réouverture de l'archipel aux Occidentaux, à la révolution industrielle, au passage d'une société féodale à une société moderne. Le professeur Nishida parle d'une «complexification » de I'identification des pratiques de restauration depuis l'entrée dans l'ère Meiji, alors que le professeur Yagasaki nous dit qu'à la même période le shikinen-zōtai devient 


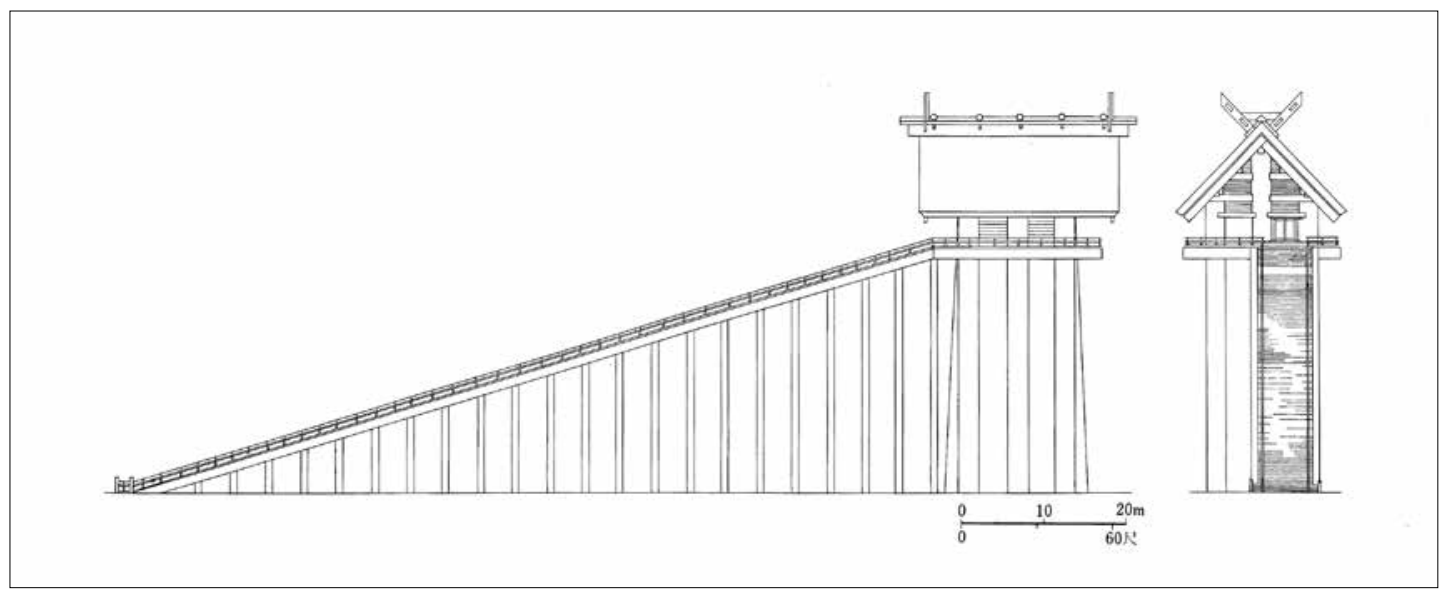

une pratique spécifique au sanctuaire $d^{\prime}$ Ise. Pouvezvous nous en dire plus sur la « marginalisation » du shikinen-zōtai et sur les modifications des pratiques de restauration à cette période charnière?

5b. Dessin restituant l'ancien honden (pavillon majeur cultuel) du grand sanctuaire shintō Izumo-taisha, tiré de Nihon kenchiku-shi zushū [Atlas illustré de l'histoire de l'architecture japonaise], Tōkyō, Shōkokusha, 1980.

- Nishida Masatsugu. Récemment, plusieurs jeunes historiens japonais ${ }^{16}$ ont à nouveau posé cette question et ils sont arrivés à des conclusions intéressantes. En bref, le gouvernement formé en 1868 a fait de la religion shintō une religion d'État afin de légitimer sa prise de pouvoir. Malgré des difficultés financières importantes, il engagea de grandes dépenses pour construire des bâtiments publics appropriés à la modernisation qu'il ambitionnait. Ces dépenses furent compensées par une large baisse des budgets alloués aux sanctuaires shintō, alors que le shintō venait d'être désigné comme religion d'État. Ainsi, en 1871, le ministère des Finances notifia-t-il aux sanctuaires shintō une obligation de diminution de superficie de leurs édifices, allant jusqu'à prévoir un contrôle de leur forme architecturale. Si les grands sanctuaires avaient respecté cette notification, ils auraient été, au moment de l'exécution de leurs shikinen-zōtai, contraints de modifier la forme de leurs pavillons, ce qui aurait induit la perte des formes architecturales anciennes. À la suite de la contestation énergique du sanctuaire d'Izumo, I'un des plus puissants du Japon (fig. 5a-b), une nouvelle notification fut édictée en 1875. Celle-ci indiquait que les grands sanctuaires anciens devaient veiller à « conserver leur forme architecturale ancienne ». En vertu de ce principe de conservation formelle, cette notification interdit également aux grands sanctuaires d'effectuer le zōtai, c'est-à-dire de restaurer en reconstruisant. Ainsi, à partir du début de l'ère Meiji, les grands sanctuaires shintō, parmi lesquels Izumo-taisha, Kamigamo-jinja, Shimogamo-jinja, Kasuga-taisha ont-ils vu, les uns après les autres, la fin de leurs shikinenzōtai. Ils allaient donc être réparés comme l'étaient les temples bouddhiques et l'architecture domestique, c'est-à-dire selon l'autre grande méthode de restauration traditionnelle : le démantèlement, total ou partiel.

- Yoshida Kōichi. Ces deux notifications gouvernementales de 1871 et 1875 sont bien connues et essentielles dans l'histoire de l'architecture japonaise. Elles sont en réalité les premières orientations politiques modernes dans le processus d'institutionnalisation de la conservation de l'architecture ancienne au Japon. L'idée de « conservation architecturale » mise en place pour les sanctuaires shintō fut rapidement étendue à d'autres types d'architecture. En 1897, après 
avoir défini un cadre institutionnel pour les questions de conservation, le gouvernement édicta une " loi sur la conservation des sanctuaires et des temples anciens", qui consistait en deux éléments majeurs : la désignation des bâtiments à protéger et la réparation de ces bâtiments par subvention de l'État; ce qui donna un cadre institutionnel aux réparations par démantèlement.

D’après les recherches récentes que vient de mentionner le professeur Nishida, il apparaît en effet que les architectes restaurateurs de l'époque, diplômés de l'Université impériale, se soient très vite appliqués à réglementer et à structurer cette pratique de démantèlement pour en faire une chaîne opératoire scientifique applicable à tout édifice classé sur la liste du patrimoine architectural à préserver. Dans le démantèlement tel qu'il était pratiqué au début du $\mathrm{XX}$ e siècle, on observe de très nombreuses réminiscences de la pratique prémoderne. Toutefois un point essentiel avait changé. Du Moyen Âge (1185-1600) à la fin de I'Époque moderne (1600-1868), le démantèlement semble avoir eu pour objectif principal la modification de l'aménagement des édifices. Au contraire, au début de l'ère Meiji, son objectif devint la conservation de la forme. Dans ce changement on peut identifier deux dynamiques : I'absorption, dans le démantèlement, du zōtai mis à mort par le pouvoir politique ; et l'apparition d'une volonté de préservation copiée sur l'Occident, avec les concepts qui l'accompagnent. C'est sans doute là le plus surprenant. Dans la pratique du démantèlement réformée à l'ère Meiji, l'influence européenne, voire française, se perçoit clairement, notamment dans la notion de "restauration stylistique » telle que l'avait pensé Eugène Viollet-le-Duc. Souvenons-nous que les architectes qui ont dirigé les grands travaux de restauration au début de l'ère Meiji, Itō Chūta (1867-1954) et Sekino Tadashi (1868-1935) notamment, ont pu consulter le Dictionnaire de l'architecture médiévale ${ }^{17}$ de Viollet-le-Duc dont une copie était conservée à la bibliothèque de l'Université impériale. Et au Japon, contrairement à ce que l'on a vu en Europe, cette idée de restauration stylistique, de retour vers un état idéal, n'était absolument pas critiquée.

Le démantèlement tel qu'on le pratique aujourd'hui est donc un procédé dont la stratigraphie est relativement complexe. Il est à la fois un héritage légitime de la longue histoire de la transmission architecturale au Japon, mêlant le zōtai et le zuihashūri (« réparation occasionnelle »), et le résultat de l'adaptation d'idées patrimoniales venues d'Occident. En cela, ce modèle représente, dans sa globalité, la version moderne de la tradition architecturale japonaise. Et la confusion dont il était question au début de notre conversation apparaît maintenant assez logique.

- Jean-Sébastien Cluzel. Vous venez d'évoquer Viollet-le-Duc et de sous-entendre qu'il ait pu avoir une influence au Japon. Or aujourd'hui, dans l'esprit des Européens, une restauration à la Viollet-le-Duc ressemblerait plutôt à une restitution, c'est-à-dire au retour vers un état antérieur, qui indubitablement renferme une part d'idéal. Pensez-vous qu'en matière de restauration, les pratiques japonaises modernes et contemporaines correspondent à la définition qu'en donnait Viollet-le-Duc?

- Yoshida Kōichi. Au début de l'ère Meiji, c'est certain. À cette époque, le démantèlement était globalement interprété comme une pratique permettant de retrouver l'état d'origine d'un bâtiment. Sekino Tadashi, que nous venons d'évoquer, employa cette méthode pour restaurer le pavillon principal du temple bouddhique Shin-Yakushi-ji, fondé à l'époque de Nara (710-794). Ce chantier débuta peu après l'édiction de la « loi sur la conservation des sanctuaires et des temples anciens " de 1897 et permit de remettre le pavillon dans son état d'origine, supprimant quasiment toutes les parties qui avaient été ajoutées ou modifiées au cours du temps. Comme les restaurations de Viollet-le-Duc, celle-ci fut critiquée par les contemporains de Sekino. La plupart d'entre eux soulignaient l'étrangeté du pavillon 
totalement modifié et manifestaient un grand scepticisme quant à la véracité d'une telle « restitution ». Le critique littéraire Takayama Chogyū (Rinjirō, 1871-1902), plus raisonnable, interrogea quant à lui la disparition des détails architecturaux du Moyen Âge ${ }^{18}$. Quelques années plus tard, dans le numéro de la revue Rekishichiri [Histoire-Géographie] de septembre 1901, Tsuji Zen'nosuke (1877-1955), spécialiste de l'histoire du bouddhisme, publia les orientations du protocole suivi pour effectuer cette restauration ${ }^{19}$ :

- Si la construction présente des ajouts postérieurs qui dégradent le style architectural et si l'état d'origine est évident : restituer.

- Pour les cas douteux, quand on ne peut pas distinguer un éventuel ajout de l'état d'origine : laisser en l'état, en espérant la réalisation de prochaines études.

- Dans le cas d'un ajout postérieur évident, mais sans information sur l'état d'origine, il faut imaginer une restitution.

- Les ajouts d'une grande valeur artistique et historique doivent être conservés. Pour consolider les structures des édifices, les techniques actuelles sont autorisées si elles n'altèrent ni la forme, ni l'apparence stylistique. Mais dans ce cas, il faut optimiser la réutilisation des membres de la structure d'origine et des matériaux anciens, en gardant leurs couleurs anciennes.

Ce bilan résume les mesures appliquées par les ingénieurs lors de la restauration de Shin-Yakushi-ji. Tout cela est moins radical que l'on veut parfois le croire. Excepté le peu de valeur accordée aux membres de la structure, ces lignes de conduite sont relativement similaires à celles énoncées dans la charte de Venise (1964) qui sont recommandées aujourd'hui.

D'une manière générale, les travaux de restauration postérieurs à ceux du Shin-Yakushi-ji n'ont jamais visé une restitution complète de l'état d'origine, mais ont plutôt permis de conserver les parties ajoutées et modifiées au fil du temps. De même, à partir de l'ère Meiji, les restaurations qui ont conduit à un démantèlement sont assez rares. D'ailleurs, il est évident que le Japon ne s'est pas engagé dans une application systématique du démantèlement visant à restituer l'état d'origine des édifices.

Finalement, je crois que l'on peut dire que depuis la fin du XIX ${ }^{\mathrm{e}}$ siècle, I'histoire de la restauration au Japon n'est pas tellement différente de celle de l'Europe, oscillant entre les théories de Viollet-le-Duc, John Ruskin, Camillo Boito et Gustavo Giovannoni.

- Jean-Sébastien Cluzel. Il y aurait donc une sorte de stigmatisation des pratiques de conservation japonaises, par l'apposition d'une étiquette "zōtai », étiquette tirée d'une tradition révolue. L'étrangeté résiderait plutôt dans cette volonté forte -à la fois occidentale et japonaise - d'affirmer des particularités identitaires, alors que les pratiques japonaises sont en partie empruntées à l'Occident et, pourrions-nous même dire, qu'elles répondent aux exigences des normes occidentales de la conservation.

- Yoshida Kōichi. L'intérêt européen pour le skikinen-zōtai est relativement récent. Bien sûr, cette pratique est mentionnée par Basil Hall Chamberlain dans son ouvrage Japanese Things ${ }^{20}$ publié en 1890. Elle est également décrite dans le journal de voyage du botaniste anglais Richard Gordon Smith ${ }^{21}$, le 5 octobre 1904, date à laquelle il visite le sanctuaire d'Ise. Curieusement, Smith écrit que " la forme des pavillons est fidèle à celle du IVe siècle avant Jésus-Christ, jusque dans leurs moindres détails, malgré les reconstructions effectuées tous les vingt ans ». Lafcadio Hearn, écrivain irlandais, remarque lui aussi, dans son ouvrage de $1904^{22}$, que le sanctuaire d'Ise doit être reconstruit tous les vingt ans selon les coutumes antiques. Mais avant la fin du XIXe et le début du XXe siècle, on ne trouve pratiquement aucune mention du shikinen-sengū dans les textes occidentaux. Ni dans l'História do Japão du jésuite portugais Luís Fróis ${ }^{23}$, ni dans Les Jésuites au Japon : relation missionnaire d'Alessandro $V_{\text {Valignano }}{ }^{24}$, ni dans les correspondances de François Xavier ${ }^{25}$, le shikinen-sengū n'est 
mentionné. Ce qui semble assez logique si l'on considère qu'aucun rituel n’a été exécuté au cours de leurs séjours au Japon, d'ailleurs aucun d'eux ne s'est rendu à Ise. Dans les documents postérieurs, tel Le Puissant royaume du Japon de François Caron ${ }^{26}$, dans le journal d'Engelbert Kaempfer ${ }^{27}$, ou encore dans Voyage au Japon, exécuté pendant les années 1823 à 1830 de Philipp Franz von Sieboldd ${ }^{28}$, le shikinen-sengū n'est toujours pas mentionné. En somme, ce rituel a commencé à intéresser les Européens au cours de l'ère Meiji.

Par contre, les descriptions sur le caractère non-permanent, sur la facilité du transfert des édifices japonais sont assez nombreuses, par exemple dans I'ouvrage de Bernardino de Avila Giróndans, Relación del Reino de Nippon a que llaman corruptamente Jappon, écrit entre 1598 et 1619, mais publié en 1883 ou encore dans celui de Johannes G. F. O. Fischer intitulé Bijdrage tot de kennis van het Japansche rijk [Contribution à la connaissance de l'État du Japon] publié en 1833. Quelques années plus tard, dans Le Japon de nos jours, ouvrage publié en 1877, le juriste français Georges Bousquet écrit que dans l'archipel, même les bâtiments les plus importants sont construits en bois, le matériau le plus modeste qui soit, et il ajoute que ce matériau ne permet pas, de par sa nature, d'offrir à l'architecture japonaise la pérennité : élément indissociable d'une œuvre architecturale!

Ce genre de remarques sur le caractère non-pérenne et provisoire de l'architecture ne peut pas se limiter à l'architecture japonaise, il concerne l'architecture asiatique en général. Dans le Livre des merveilles, Marco Polo parle d'un " palais somptueux ${ }^{29}$ " en bambou transportable qui est démantelé et stocké dans la ville de Chandu. Un missionnaire français sinologue, Joseph-Marie Calley, qui fut chargé, entre 1857 et 1859, d'une chronique sur l'architecture chinoise pour la Revue générale de l'architecture et des travaux publics, a décrit longuement le caractère non pérenne de l'architecture de l'empire du milieu.

D'ailleurs, Viollet-le-Duc s'inscrit dans cette même ligne. Dans son Dictionnaire raisonné de l'architecture française du XIe au XVI siècle, il écrit :

En Asie, autrefois comme aujourd'hui, lorsqu'un temple ou un palais subissait les dégradations du temps, on en élevait ou l'on en élève un autre à côté.

On ne détruit pas pour cela l'ancien édifice ; on l'abandonne à l'action des siècles, qui s'en emparent comme d'une chose qui leur appartient, pour la ronger peu à peu ${ }^{30}$.

Dans un autre ouvrage, Histoire de l'habitation humaine depuis les temps préhistoriques jusqu'à nos jours ${ }^{31}$, Viollet-le-Duc souligne avec insistance que la restauration est une notion moderne, absente des périodes anciennes, et qui n'existe pas encore en Asie. Entre ces lignes, apparaît l'image de la fragilité de l'architecture asiatique, qui serait issue d'un renoncement laissant tout pouvoir à la nature. Ce sentiment fut bientôt largement partagé par les Européens qui trouvèrent dans le shikinen-zōtai une expression parfaite de cette fragilité caractéristique de l'architecture japonaise

- Jean-Sébastien Cluzel. Cette étiquette de la reconstruction systématique, qu'on la nomme zōtai ou "démantèlement ", représente, pour les Occidentaux et depuis la fin du XIXe siècle seulement, l'identité patrimoniale japonaise. Si une telle étiquette correspond à une certaine réalité, vous laissez entendre que l'image renvoyée est en quelque sorte pervertie par une vision matérialiste très éloignée de la culture japonaise, c'est-à-dire une vision occidentale. Peut-on dire que cette étiquette fut « acceptée » pour favoriser le dialogue avec l'Occident, mais que l'essence de l'architecture japonaise est ailleurs?

- Yoshida Kōichi. Il me semble que la convention ratifiée par le Japon en 1992 - « convention concernant la protection du patrimoine mondial, culturel et nature ${ }^{32}$ » de I'UNESCO, qui hérite de la notion d'authenticité de la charte de Venise - est le point de départ 
de la stigmatisation des pratiques de conservation japonaises. Or cette charte développe une vision très théorique et académique de la conservation. Elle est régie par une moralité ruskinienne plutôt que par le caractère pratique d'un George Gilbert Scott. Certes, la charte de Venise est une déclaration de haute tenue, mais elle est irréaliste, même pour les Occidentaux, sa mise en pratique est impossible. En 1994, la Conférence de Nara sur l'authenticité33 a permis de grandes avancées, notamment en retrouvant l'esprit modéré de la charte d'Athènes (1931) qui reflète davantage la diversité culturelle des sociétés.

- Nishida Masatsugu. Pour ma part, il me semble que le Japon s'est longtemps laissé enfermer dans une discussion matérialiste, alors que, comme l'a laissé entendre le professeur Yoshida, l'essence de l'architecture japonaise est ailleurs, dans le provisoire, le temporaire, l'éphémère et même l'immatériel.

Ici, je ne veux pas dénoncer quelque méfait de l'occidentalisation de la culture japonaise. Au contraire, je pense que la confrontation des cultures japonaise et occidentale a facilité la révélation de l'essentialité de la première. En ce sens, l'ère Meiji a, me-semble-t-il, permis de mieux comprendre, voire même de découvrir la singularité culturelle du Japon. C'est à cette époque que l'architecture japonaise a été comparée à l'architecture occidentale, non seulement en confrontant des bâtiments, mais aussi les façons d'appréhender ces bâtiments.

Vous le savez, avant la réouverture du Japon, la langue japonaise ne disposait pas de terme équivalent à la notion occidentale d'architecture. La création de cet équivalent - avec toutes ses hésitations - eut lieu au début de l'ère Meiji. L'architecture fait appel à des concepts appartenant à la culture européenne, concepts qui explicitent les mœurs, «I'habiter », et le rapport créé entre les hommes et les bâtiments qu'ils édifient. Ainsi la traduction du terme architecture en japonais n'est pas une simple transposition, elle est une modification de la manière de regarder les édifices et de concevoir l'acte de bâtir. Et c'est bien ce processus de mutation qu'a choisi de suivre le Japon dans la deuxième moitié du XIXe siècle. Aujourd'hui, le terme « architecture » est traduit par kenchiku 建築. Mais au début de l'ère Meiji, - je parlais d'hésitations - le ministère de l'Ingénierie du gouvernement Meiji, qui s'occupait de la construction des bâtiments publics et de la formation des futurs architectes japonais, avait d'abord opté pour le terme zōka 造家, qui possède une forte connotation technique et qui se constitue de deux caractères chinois : le premier zō/tsukuru 造 signifiant «fabriquer, construire, créer »; le second ie 家 signifiant... « maison »! Or il est évident que les Japonais du début de l'ère Meiji savaient que les Occidentaux ne bâtissaient pas que des maisons et ils savaient aussi que l'architecture occidentale ne traitait pas uniquement de construction. Cependant, c'est cet assemblage de caractères qu'ils ont choisi pour traduire le terme " architecture ». Ce choix initial est une piste pour recouvrer l'idée que se faisaient les Japonais de leur propre architecture au moment de la réouverture du pays : pour eux, la « maison », ie 家, représentait beaucoup plus qu'un bâtiment destiné à être habité ! D'ailleurs cette perception large de la maison perdure. De nos jours, les personnes âgées ou les habitants des villages de campagne utilisent encore le terme ie 家 pour désigner n'importe quel type de bâtiment.

Pour comprendre les différences de sens et de perception entre l'Europe et le Japon, pour en saisir l'évolution, d'un côté comme de l'autre, il me semble que les dictionnaires bilingues anciens sont des sources incomparables. Dans le dictionnaire latin-japonais édité par le jésuite Ambrogio Calepino en $1595^{34}$ - premier dictionnaire du vocabulaire occidental publié au Japon - les termes zousaku 造作, zōei 造営 et konryū 建立 sont proposés pour traduire aedificum, tandis que ie wo tatsuru イエヨタツル et konryū suru コンリュウスル, c'est-à-dire « construire une maison » ou " bâtir », sont des expressions proposées pour traduire architectura. Un peu plus tard, entre la deuxième moitié du XVIII ${ }^{e}$ siècle et la première moitié du XIXe siècle, un dictionnaire hollandais-japonais ${ }^{35}$ est publié, dans lequel les termes « bâtir » et « construire » 
sont traduits par ie wo tateru 家Э建テル, ce qui signifie littéralement «bâtir une maison ». Et pour le terme « architecture », ce dictionnaire donne ie wo tateru jutsu 家Э建テル術, ce qui signifie « l'art de bâtir une maison ». C'est donc seulement vers la fin de l'époque d'Edo, dans la deuxième moitié du XIXe siècle, que des termes comme zōka 造家 et kenchiku 建築 ont commencé à être utilisés pour traduire le terme " architecture ».

Si l'on souhaite s'interroger sur les fondements des concepts de l'architecture japonaise, il faut s'intéresser aux textes de l'Antiquité. Ceux-ci font apparaître, pour le terme «maison», $i e$ 家, une dimension spatiale complètement étrangère à la notion occidentale d'architecture. Ici, le mot «maison », ie イ工, n'implique pas une construction matérielle. D'après des études lexicographiques et comparatives conduites sur les chants réunis dans le Man'yōshū [Recueil des mille feuilles], le Kojiki [Chroniques des faits anciens] et le Nihon shoki [Chroniques du Japon], ouvrages compilés à l'époque de Nara, au VIII' siècle, il apparaît que le terme ie イエ était utilisé pour désigner un emplacement, voire une étendue, dans laquelle se trouvait une maison en tant qu'espace de résidence particulière, plutôt qu'en tant qu'élément bâti. Le Kojiki [Chroniques des faits anciens] contient le plus ancien poème japonais mentionnant une maison. La divinité Susano-o no Mikoto y dit ceci :

Yakumo tatsu / Izumoyaegaki / Tsumagomi ni / Yaegaki tsukuru / Sono Yaegaki wo

八雲立つ 出雲八重垣 妻籠みに 八重垣つくる その八重垣を

Huit couches de nuages s'élèvent

Les nuages forment huit rangées de haies.

Pour installer ma compagne

Huit rangées de haies se sont formées.

Ah! Ces huit rangées de haies!

Dans ce poème, rien ne suggère l'existence de la maison en tant que construction matérielle. Fonder une maison est décrit comme l'établissement de " clôtures», kaki カキ, terme qui peut aussi désigner une demeure. De plus, une telle organisation, à clôtures multiples, rappelle immédiatement les quadruples palissades du sanctuaire d'Ise...

À travers cette petite analyse lexicographique, il est facile de comprendre que les concepts qui régissent les termes qui nous intéressent dans cette discussion - architecture,

6. Photographie du Daijō-kyū, sur la droite se dresse I'un des deux pavillons jumeaux, le sukiden, dont le pendant est le yukiden; sur la gauche on voit la toiture du kairyūden. Ce complexe religieux a été construit à l'intérieur du Palais impérial de Tōkyō pour une cérémonie d'intronisation de l'empereur Naruhito, 2019.

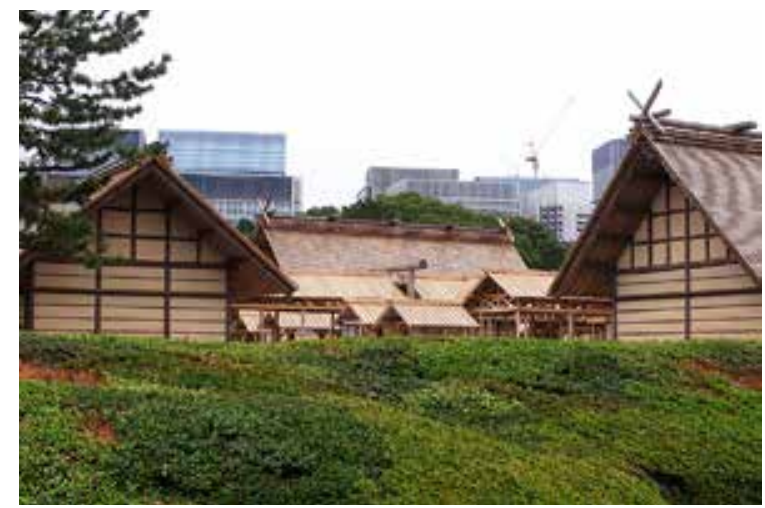
construction, maison, mais aussi reconstruction, restauration ou rénovation - se fondent sur des traditions fort différentes. Les églises chrétiennes par exemple se sont forgées sur des modèles précis qui visent à représenter la « maison de Dieu ». Leurs formes et leurs espaces intérieurs sont bien distincts de ceux des maisons des hommes. Elles ont été bâties comme des monuments éternels, avec des matériaux, des espaces et des formes qui font échos à la liturgie. En revanche, au Japon, les sanctuaires ressemblent aux maisons des hommes. Au lieu d'inventer une typologie particulière pour « la maison de Dieu ", les Japonais ont construit une maison pour Dieu. Ainsi, avant l'influence chinoise et I'importation du bouddhisme, il semble qu'il y ait eu peu de différences entre une maison 
ordinaire, un palais et un sanctuaire. Et ces trois types d'architecture sont restés beaucoup plus homogènes qu'en Occident. Dans ce contexte, il est tout à fait logique que les études modernes en histoire de l'architecture japonaise se soient intéressées à ces trois typologies sans vraiment les distinguer. Les historiens, les archéologues et les ethnologues cherchaient tous l'origine de la forme, de la disposition du plan et de l'organisation spatiale de la «maison » dans l'architecture shintō, palatiale ou domestique.

Aujourd'hui encore, les historiens, en s'appuyant sur les Chroniques des faits anciens, s'accordent à voir cette origine formelle dans les édifices du grand sanctuaire d'Izumo. Dans le Kojiki, la divinité Ōkuninushi no ōkami, qui depuis a élu résidence au sanctuaire d'Izumo, demande aux hommes de lui construire un palais identique à celui de leur empereur, en compensation du pays qu'il leur concède. Ce palais est le sanctuaire d'lzumo. Et pour les Japonais, ce sanctuaire, ayant suivi le shikinen-sengū depuis sa fondation au VIII siècle jusqu'au XVII siècle, témoigne de cette forme palatiale antique. Le célèbre historien de l'architecture japonaise, Ōta Hirotarō, écrit dans son Introduction à l'histoire de l'architecture japonaise ${ }^{36}$ :

Quant aux dimensions concrètes des bâtiments du palais, le manque de sources écrites ne nous permet pas de les connaître. Par contre, les édifices du sanctuaire shintō construits sur le modèle du palais de cette époque nous en transmettent la forme. Ce sanctuaire shintō nous permet donc d'imaginer cette forme.

Cette interprétation, qui fait consensus, est induite par la proximité des architectures palatiale et domestique. Si cette proximité est spécifique au Japon, les thèmes de recherche sont également contraints par une appréhension propre à cette culture architecturale, imprégnée du shikinen-sengū : il ne s'agit pas de récupérer des vestiges du palais, d'en conserver la matérialité, mais d'en restituer la forme, une forme qui est finalement immatérielle.

- Jean-Sébastien Cluzel. Vos propos et l'actualité de la famille impériale me rappellent un palais très particulier, le Daijō-kyū, somptueuse construction, dont la vie se résume à quelques jours. Ce palais ou cette maison de Dieu, entourée de plusieurs rangées de clôtures, se compose de trois bâtiments principaux qui permettent à la déesse Amaterasu de retrouver le futur empereur, sur le chemin de son avènement, pour partager avec lui, au cours d'une nuit seulement, un repas et un bain. Ce palais est sans doute le plus éphémère de l'archipel. L'empereur n'y entre qu'une fois dans sa vie de mortel. Construit avec des matériaux neufs, il est monumental par sa taille, son lieu de construction n'est pas fixe : pour l'avènement de l'empereur Hirohito il avait été érigé au sein du palais impérial de Kyōto, pour l'avènement de l'empereur Naruhito il a été érigé à Tōkyō (fig. 6), et il vient d'être démantelé. Peut-on considérer que ce palais, entre la maison du dieu et le palais impérial, est un édifice représentatif des spécificités japonaises dont nous parlons?

- Nishida Masatsugu. Bien entendu, mais il faut faire attention. Si le Daijō-kyū possède ces spécificités japonaises, il est périlleux d'en faire un exemple représentatif. Cela ouvrirait la porte à des raccourcis faciles, similaires à ceux qui ont pu être pris en portant notre attention sur le shikinen-sengū au sanctuaire d'Ise. De plus, choisir d'illustrer ces particularités japonaises à partir d'édifices religieux
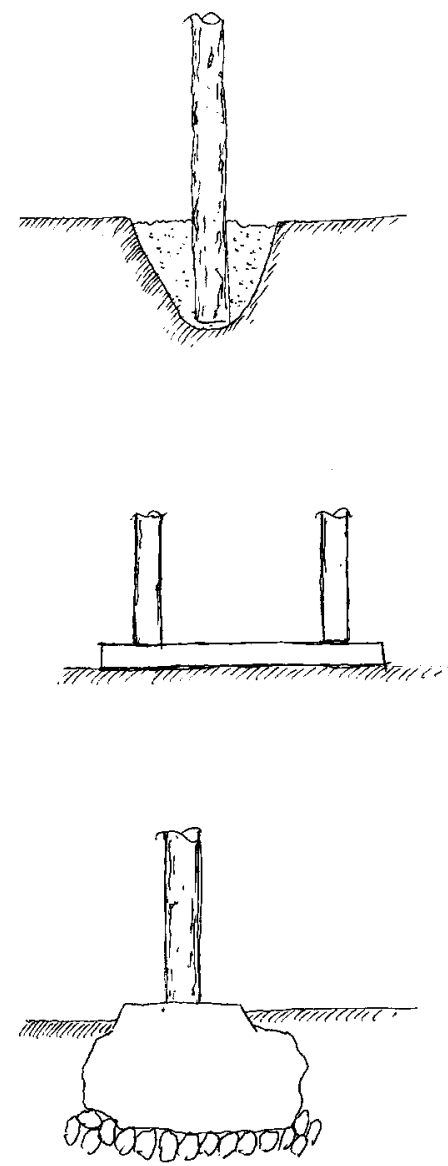

7. Du haut vers le bas, fondation de type hottate, fondation de type dodai, fondation de type sosekidate. 


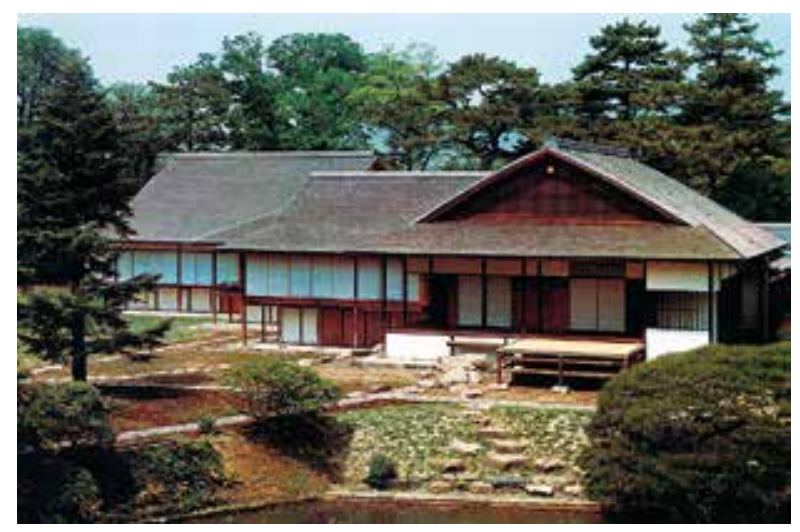

8a. Photographie de la façade principale, en vol d'oies sauvages, de la villa Katsura, Kyōto, 1983.

8b-d Plans géométraux de Katsura-rikyū montrant trois étapes de développement : $1^{\text {ère }}$ campagne vers 1616 [b] ; $2^{\mathrm{e}}$ campagne vers 1641 [c] ; $3^{\mathrm{e}}$ campagne vers 1663 [d]. Images tirées de Saito Hidetoshi, Katsura-rikyū, Tōkyō, Shōgakkan, 1990, p. 49-50. inclus dans des rituels qui sont eux-mêmes liés à la légitimation du pouvoir de la famille impériale accroît les risques de confusion entre un discours nationaliste ou impérialiste et une réalité historique.

Pour ma part, je pense que cette immatérialité, cette impermanence, ce côté transitoire se retrouvent dans toute l'architecture japonaise, et ces particularités me semblent être un caractère plus prégnant et plus significatif pour l'architecture japonaise que ne le sont les concepts plus matérialistes. Aussi, pour bien comprendre ces particularités, il faut élargir le corpus architectural et regarder aussi des palais, des villas ou encore des pavillons de thé...

- Yagasaki Zentarō. Je suis du même avis que le professeur Nishida. Pour discuter le caractère transitoire et non pérenne de l'architecture japonaise, j'aimerais vous faire partager quelques observations sur les techniques de fondation. Dans la tradition

japonaise, c'est-à-dire pour ce qui concerne l'architecture des périodes historiques, les fondations des édifices en bois peuvent être mises en œuvre selon trois méthodes différentes. La première, hottate 掘立, consiste à creuser un puits dans le sol, à y placer la base du poteau de bois, puis à tasser la terre remblayée. La deuxième méthode, dodai 土台, consiste à poser sur la terre un socle fait de bois équarris horizontaux sur lequel un poteau est ensuite fixé avec des ferrures. La troisième méthode, sosekidate 礎石建 $て$, consiste à donner pour base au poteau une pierre qui repose directement sur le sol (fig. 7).

Les édifices réalisés selon la méthode hottate s'affaissent rapidement, en raison du pourrissement des parties enfouies des poteaux. Cette méthode est donc essentiellement utilisée pour des bâtiments légers qui ne sont pas construits pour être pérennes. La deuxième méthode, dodai, présente le même inconvénient lié au pourrissement, puisque le socle en bois repose lui aussi sur la terre. Par contre, cette technique permet de déplacer facilement les édifices puisque les bases des poteaux ne sont pas ancrées dans le sol, mais simplement posées sur un socle en bois équarri. Ces deux premières méthodes - hottate et dodai - sont relativement simples et rapides à mettre en œuvre pour ériger des poteaux, mais elles conviennent surtout à des constructions provisoires ou mobiles; elles sont aussi les plus anciennes méthodes de construction dans l'archipel nippon, déjà utilisées pour les premiers édifices préhistoriques.

À l'inverse, les pierres de fondation non enfouies mises en œuvre dans le sosekidate préservent les poteaux des remontées d'humidité. Cette méthode est donc préférable pour les constructions lourdes et pérennes, et se rapproche des techniques actuelles utilisées dans les constructions en bois où les poteaux se dressent à partir des socles en bois équarri posés sur des fondations en béton qui sont plus hautes que le niveau du sol. Importée de Chine en même temps que le bouddhisme au milieu du $\mathrm{VI}^{\mathrm{e}}$ siècle, cette troisième méthode, inconnue jusqu'alors dans l'archipel, a d'abord été mise en œuvre dans les temples bouddhiques. Elle a très vite bouleversé les anciennes pratiques japonaises et fut largement développée dans l'architecture monumentale. Ces quelques remarques sur les différentes techniques utilisées 

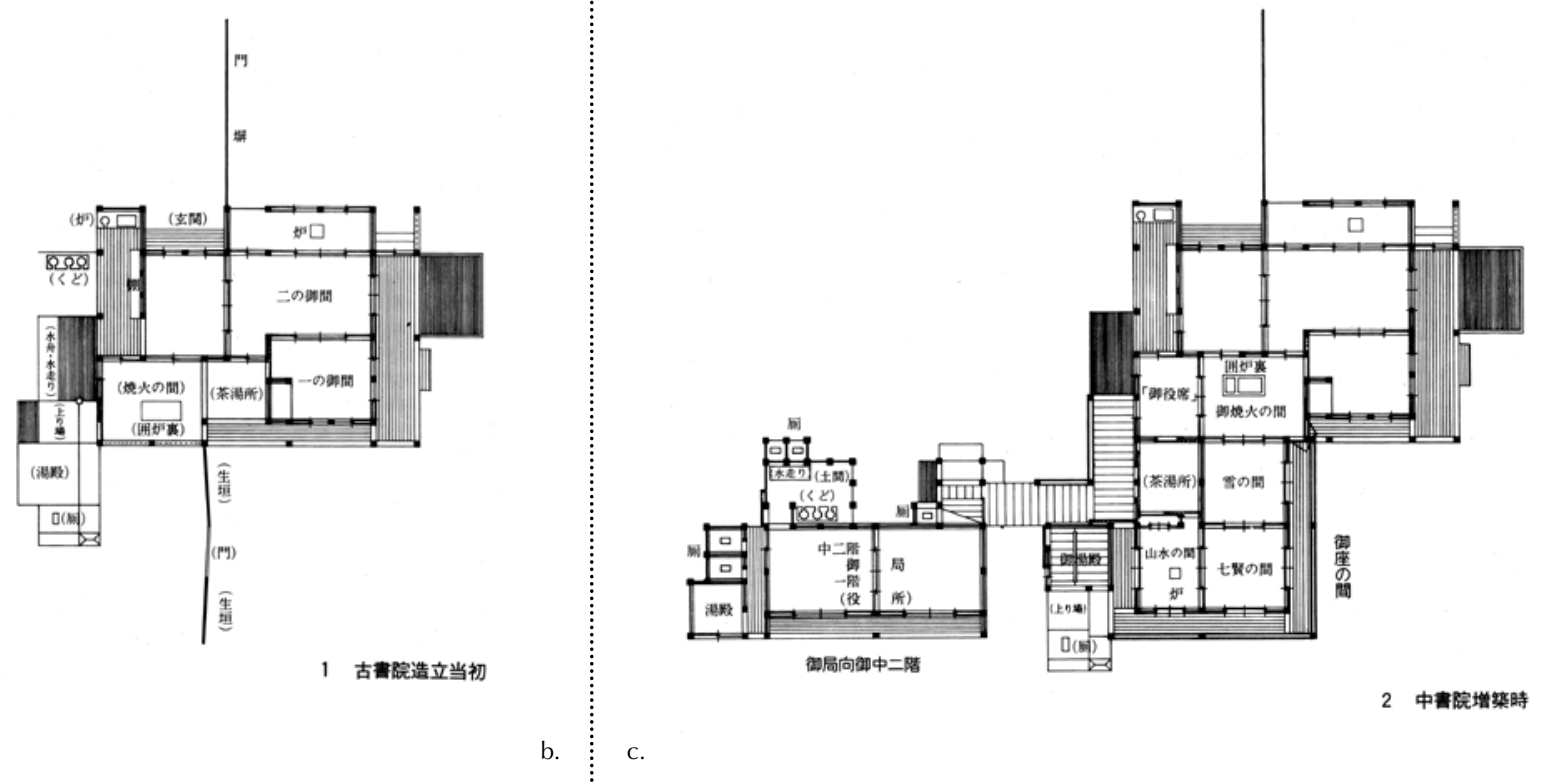

d.

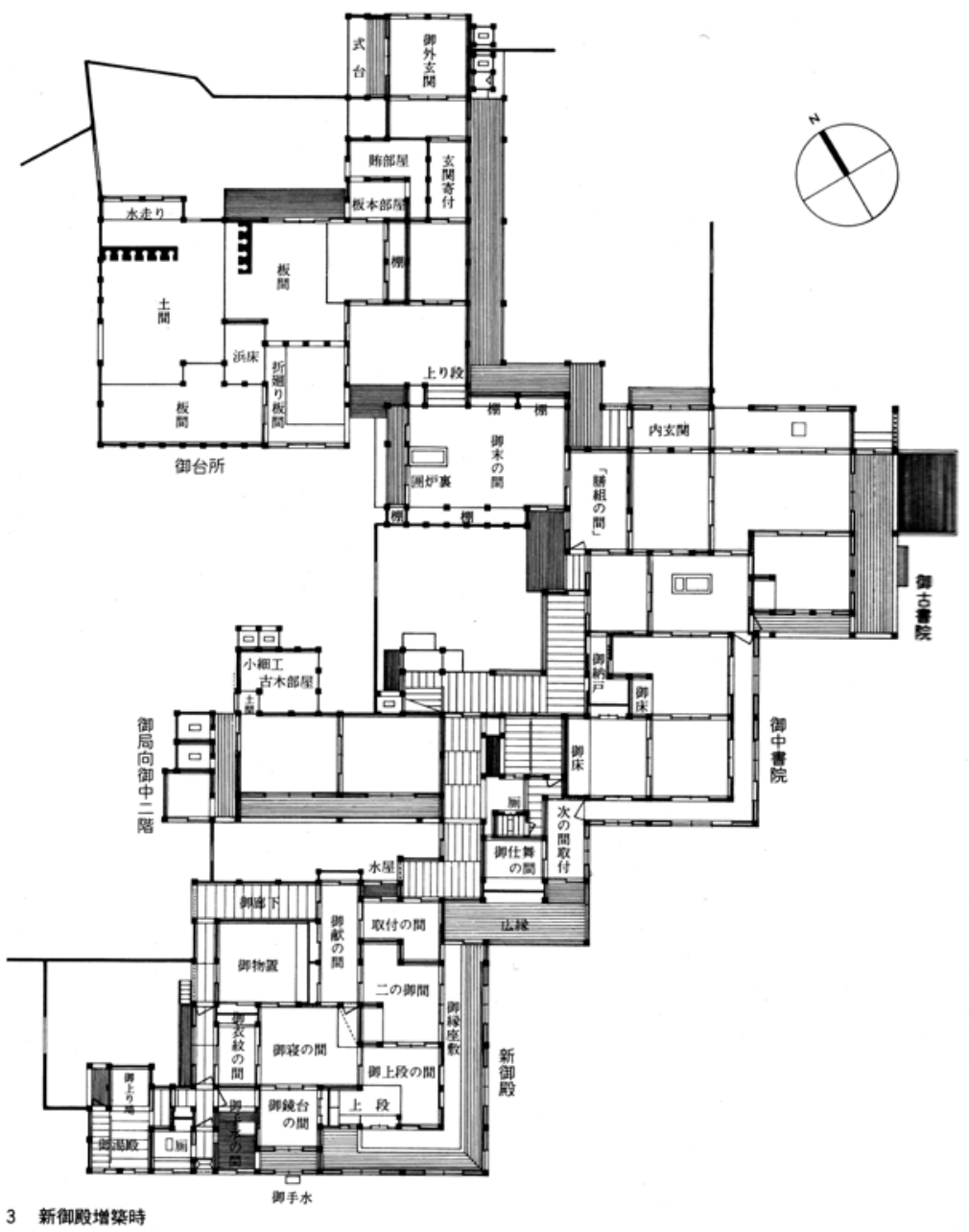



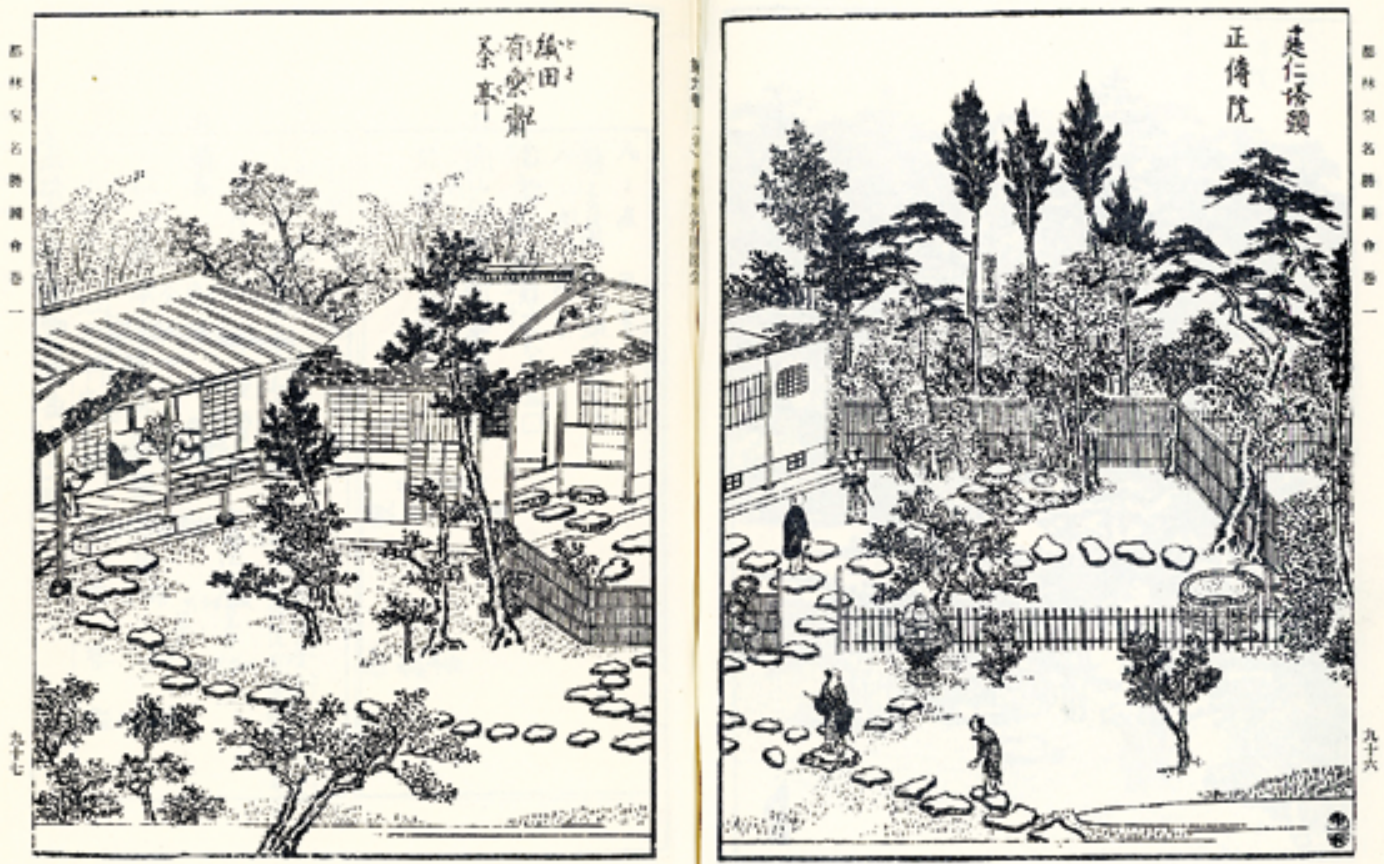

9a. Pièce de thé Jo-an dans l'enceinte du Shōden-in au cœur du monastère Kennin-ji, à Kyōto, tiré de Miyako Rinsen Meishō Zue, 1799, vol. 1-bis, fol. 18 au Japon pour dresser les poteaux permettent de comprendre que l'architecture japonaise était originellement précaire, provisoire et déplaçable ; ainsi, I'architecture japonaise eut, semble-t-il dès l'origine, ce caractère transitoire.

J'aimerais maintenant vous proposer quelques remarques sur des édifices japonais fort connus et souvent admirés comme les fleurons de l'architecture nipponne. L'un des plus célèbres, la villa impériale Katsura-rikyū (fig. 8a), présente un plan en forme d'escalier que l'on décrit habituellement comme un vol d'oies sauvages. Chacune des différentes parties de l'édifice est ici rejetée à l'arrière de la précédente, de sorte que chacune d'elles jouxte le jardin sur deux côtés, offrant ainsi des vues variées sur le paysage environnant. Avoir imaginé un bâtiment pour sublimer le paysage est certes très agréable, mais l'on peut s'interroger sur le caractère pérenne de cet ensemble architectural : le concepteur pouvait-il seulement imaginer que le bâtiment serait conservé tel quel et que les générations futures le jugeraient comme une œuvre représentative de toute l'architecture japonaise ? Cela est peu probable. À l'origine se trouvait là une petite résidence, une villa construite au début du XVII siècle par le prince Toshihito (1579-1629), le premier prince du clan impérial Hachijō. Cet édifice évolua peu à peu, pendant près d'un demi-siècle, jusqu'à prendre sa forme actuelle (fig. 8b-d). Celle-ci est donc le fruit d'additions successives réalisées par les deux générations princières suivantes. Choisir d'élargir progressivement le toit, afin de raccorder les nouveaux bâtiments aux anciens, a naturellement conduit à une construction en escalier qui transforma l'édifice originel en un ensemble cohérent. Les additions ont ensuite cessé, mais il est tout à fait possible d'imaginer que de nouveaux bâtiments soient ajoutés dans le futur. Aussi, le beau motif en vol d'oies sauvages découle davantage d'une méthode d'extension de toiture et d'ajouts que d'un projet initial. 
Les pièces de thé sont un autre exemple d'architecture caractéristique du Japon. Nombre d'entre elles furent construites dans le style sōan à partir de la fin du XVle siècle. Trois sont aujourd'hui classées trésors nationaux.

Parmi elles se trouve la pièce appelée Jo-an, construite en 1618 à Kyōto, dans l'enceinte du Shōden-in au cœur du monastère Kennin-ji (fig. 9a-d). Le maître de thé qui bâtit cette pièce, Oda Nagamasu (Oda Uraku, 1547-1622), frère cadet d'Oda Nobunaga, y passa les dernières années de sa vie. La superficie de terrain occupée par le temple Kennin-ji a été grandement réduite en 1872 par ordre du gouvernement Meiji. La parcelle du Shōden-in fut alors associée à d'autres pour former un ensemble destiné à la production de thé, dont les bénéfices aidaient à maintenir la condition des geisha et des courtisanes. Dans cette nouvelle fonction, la pièce de thé Jo-an conserva sa forme initiale, quand bien même elle était en partie utilisée au sein d'une manufacture. En 1887, le bâtiment manufacturier fut transformé en salle de réception, puis il devint le Seppin Club lors de la quatrième Exposition des industries domestiques en 1895. Ici, les fonctions assignées à la pièce de thé ont changé plusieurs fois, mais sa forme est restée la même. Malgré plusieurs changements d'attribution, depuis sa première fonction, une pièce de thé, le bâtiment a survécu en gardant quasiment sa forme initiale. Mais le domaine de l'ancien monastère fut acquis en 1908 par Takejirō Ōtani. Et tandis que le quartier se transformait pour accueillir des restaurants, les bâtiments du Shōden-in furent démantelés et peut-être vendus ou détruits. Ce rachat marqua la fin de I'utilisation de la pièce de thé dans la manufacture, mais aussi une menace pour sa survie. Par miracle, elle fut achetée par la famille Mitsui qui la déplaça dans sa résidence d'Azabu, à Tōkyō, cette même année 1908. Le Jo-an fut démonté pièce par pièce et transporté de Kyōto à Tōkyō, où il retrouva sa fonction originelle. Dix-huit ans plus tard, en 1936, il fut désigné trésor national. L'année 1908, moment de la reconnaissance de sa valeur historique, marque un tournant dans la vie du Jo-an. Trente ans plus tard, en 1938, cette pièce de thé bâtie par le célèbre Oda Uraku fut à nouveau déplacée, dans la villa Jō-zan-sō de la famille Mitsui, à Ōiso, dans la préfecture de Kanagawa, afin de la préserver des destructions de la Seconde Guerre mondiale. Le Jo-an semblait avoir trouvé là un havre de paix, mais ce répit ne dura pas, puisque la famille Mitsui décida de se séparer de cette villa en 1970. La compagnie des chemins de fer de Nagoya acquit le Jo-an en 1972 et, pour mieux le conserver, le fit déplacer dans l'une de ses propriétés, où il se trouve encore aujourd'hui, dans le jardin Uraku-en aux abords du château d'Inuyama dans la préfecture d'Aichi.

Le premier pavillon de thé classé trésor national, le Tai-an, fut initialement construit sur le flanc du mont Tenno, non loin de Kyōto, et son concepteur ne serait autre que le très célèbre maître du thé Sen no Rikyū (1522-1591). Celui-ci abandonna le pavillon quand il déplaça sa résidence. Cette pièce de thé fut réinstallée au pied de cette même montagne, tout près de Kyōto, au début de l'époque d'Edo (16031868), dans un autre temple appelé Myōki-an. On suppose donc que ce pavillon fut démonté, transporté puis rebâti.

Le troisième de ces pavillons de thé ayant le titre de trésor national, le Mittan, est conservé sur son lieu d'origine. Toutefois, ce pavillon fut

9b. Pièce de thé Jo-an dans la résidence de la famille Mitsui d'Azabu à Tōkyō.

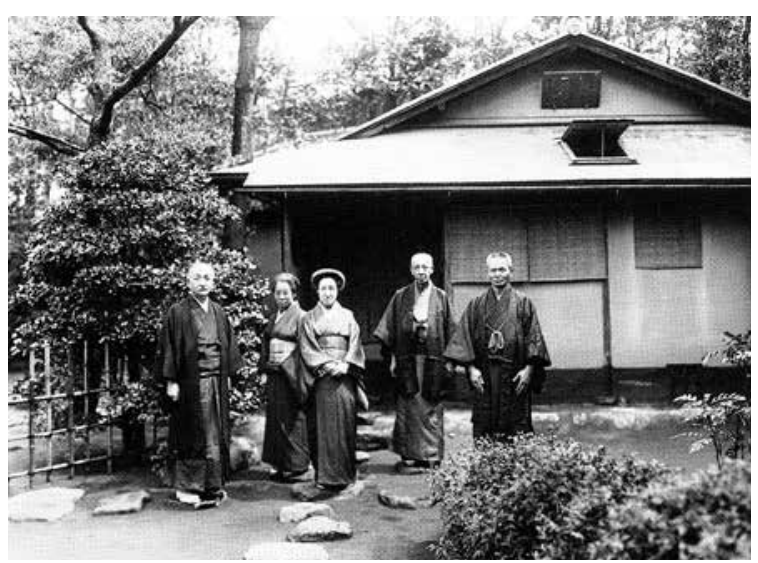


lui aussi déplacé sur une courte distance à l'intérieur de la même parcelle. Dans ce dernier cas, il est probable que l'édifice ait été transporté sans être démonté, en tirant simplement la construction vers son nouvel emplacement.

Les vies des bâtiments en bois que nous venons d'évoquer sont caractéristiques de l'architecture japonaise. Ainsi, au Japon, des ajouts et des reconstructions sont fréquemment entrepris, et il arrive que des maisons soient déplacées en étant simplement tirées, et non démontées, si tant est que la distance soit limitée ; lorsque celle-ci s'accroît, les édifices sont démontés puis transportés et remontés. Ce phénomène ne se limite pas aux constructions désignées comme patrimoniales et reconnues pour leur valeur historique, il concerne tous les types d'édifices, anciens ou récents. Les réparations et révisions constantes dont les bâtiments japonais sont l'objet leur assurent une utilisation continue dans le temps et une indépendance par rapport à leur époque de fondation. Cette continuité d'usage est essentielle, et s'il existe une attache émotionnelle au bâtiment, alors, après l'avoir démonté, il sera transporté à un autre endroit et reconstruit, puis continuera à être utilisé. L'usage de l'architecture se maintient quelle que soit la séquence des déplacements, des ajouts ou des reconstructions qu'elle subit. C'est à partir de ce mode de pensée que les structures de l'architecture japonaise ont été perfectionnées et les techniques de charpenterie adaptées. Réciproquement, l'étude de ces structures en bois montre que cette conception et cette appréhension de l'architecture fut consciente et volontaire.

Pour apprécier la valeur de l'architecture japonaise, il est donc essentiel de prendre en compte à la fois ce qui est intangible - les techniques de charpenterie - et les éléments tangibles - les structures.

La valeur de l'architecture de bois au Japon est donc attachée à

9c. Pièce de thé Jo-an lors de la reconstruction à Uraku-en. I'habileté des charpentiers, à leur capacité à transformer, voire à effacer les édifices temporairement, tout en étant à même de ramener à la

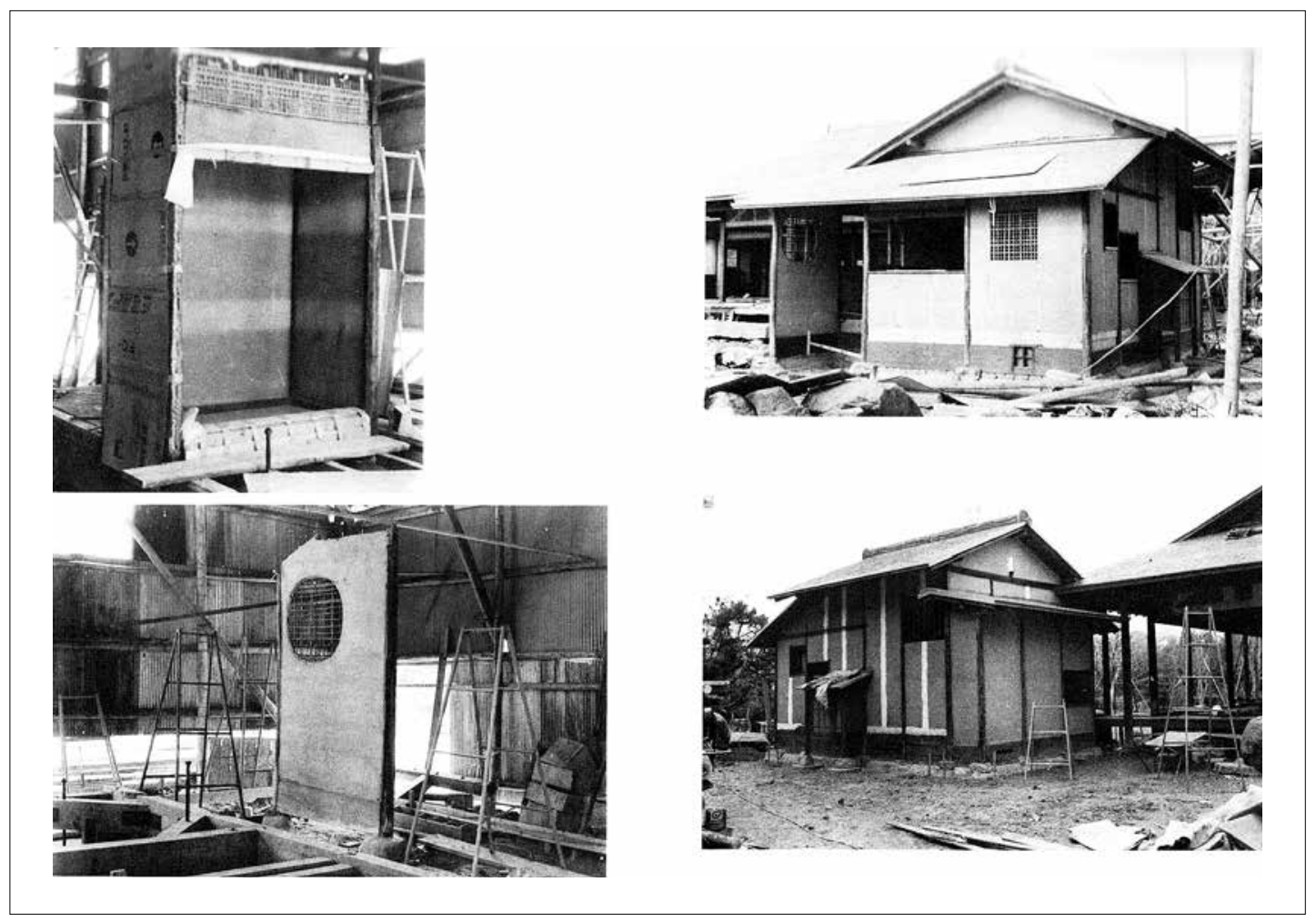


vie, dans une essentialité, la forme originelle d'un bâtiment disparu. De cette façon, I'architecture japonaise thésaurise de l'histoire, consolide sa valeur historique par la répétition séquentielle de ses déplacements, de ses ajouts, de ses reconstructions. Dans bien des cas, la date et la forme exactes de la construction d'origine sont floues, puisque le bâtiment a pu être démonté puis reconstruit de nombreuses fois. C'est en ce sens que l'architecture japonaise présente un caractère transitoire.

- Jean-Sébastien Cluzel. Vous soulignez l'importance d'une " continuité d'usage ", même si la destination de l'édifice change, et vous donnez pour exemple le pavillon de thé Jo-an. Cette voie, il est vrai, a longtemps été refusée en Europe, à l'exception des Anglo-Saxons, alors qu'elle aurait sans doute permis d'éviter à de nombreux édifices de tomber en ruine. Vous soulignez également l'importance de la conservation d'éléments tangibles - les structures - mais plus encore la

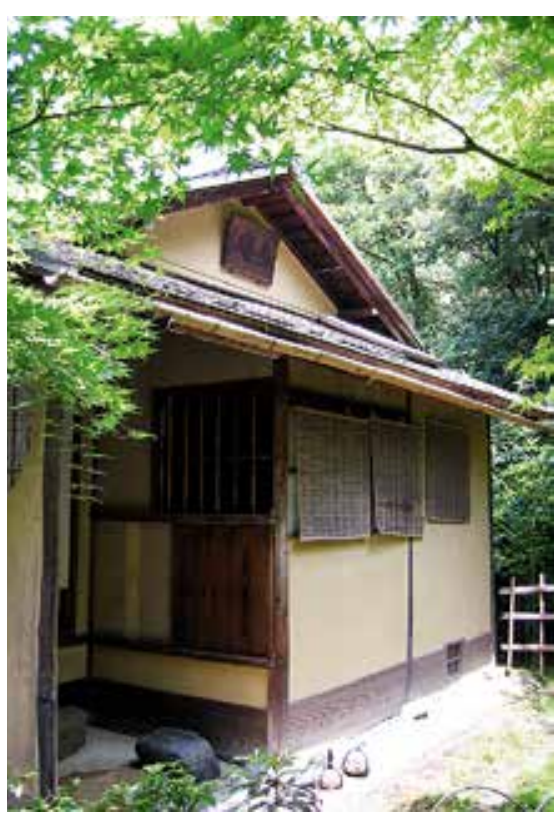

9d. Pièce de thé Jo-an, état actuel dans le jardin Uraku-en. transmission d'éléments intangibles : les techniques de charpenterie. D'un point de vue théorique, vos préconisations pour la conservation apparaissent relativement similaires aux recommandations du Getty Conservation Institute, qui encouragent les restaurations d'édifices pour lesquelles une valeur artisanale, visant à sauvegarder les savoir-faire, vient s'ajouter à la valeur patrimoniale, au sens de matérielle, de l'édifice, et équilibrer le rapport entre les deux. Alors, j'aimerais que vous nous donniez votre opinion sur un grand chantier de restauration qui a duré une dizaine d'années, celui de la chapelle principale du monastère bouddhique Tōshōdai-ji, à Nara (fig. 10a-d). Lors de cette restauration, l'édifice a été entièrement démantelé. Quand est venu le moment du remontage, les observateurs extérieurs, dont j'ai eu la chance de faire partie, ont pu voir, dans le haut de l'édifice, que le pourtour de la charpente, dans son plan horizontal, était ceinturé par des fermes occidentales, qui deviennent invisibles après le remontage de la couverture en tuile. L'utilisation de cette technique de triangulation à l'occidentale dans la restauration d'un édifice bâti entre l'an 770 et l'an 781, c'est-à-dire l'un des plus vieux édifices du Japon, vous paraît-elle choquante ? Et n'est-elle pas en contradiction avec ce que vous venez d'expliquer sur l'importance de la transmission des savoir-faire?

- Yagasaki Zentarō. La restauration de cet édifice a pris dix ans, elle a débuté en 2000 et s'est achevée en 2010. La chapelle a été démantelée dans sa totalité, pièce par pièce. Ce procédé a été utilisé parce que l'édifice prenait du ventre dans sa partie haute, les poteaux s'inclinant sous le poids de la toiture, précisément là où on a installé ce ceinturage triangulé. Les équipes de restauration se sont longtemps interrogées sur la manière dont il fallait opérer pour remédier à cette pathologie de l'édifice qui, de toute évidence, le conduisait vers l'effondrement. Devait-on remplacer les pièces défectueuses en conservant la technique ancestrale ou fallait-il donner la priorité à la conservation des matériaux d'origine ? C'est cette deuxième orientation qui a été choisie. Pour remédier à la fragilité de ces assemblages, qui n'étaient plus suffisamment solides pour supporter les contraintes de l'édifice, 

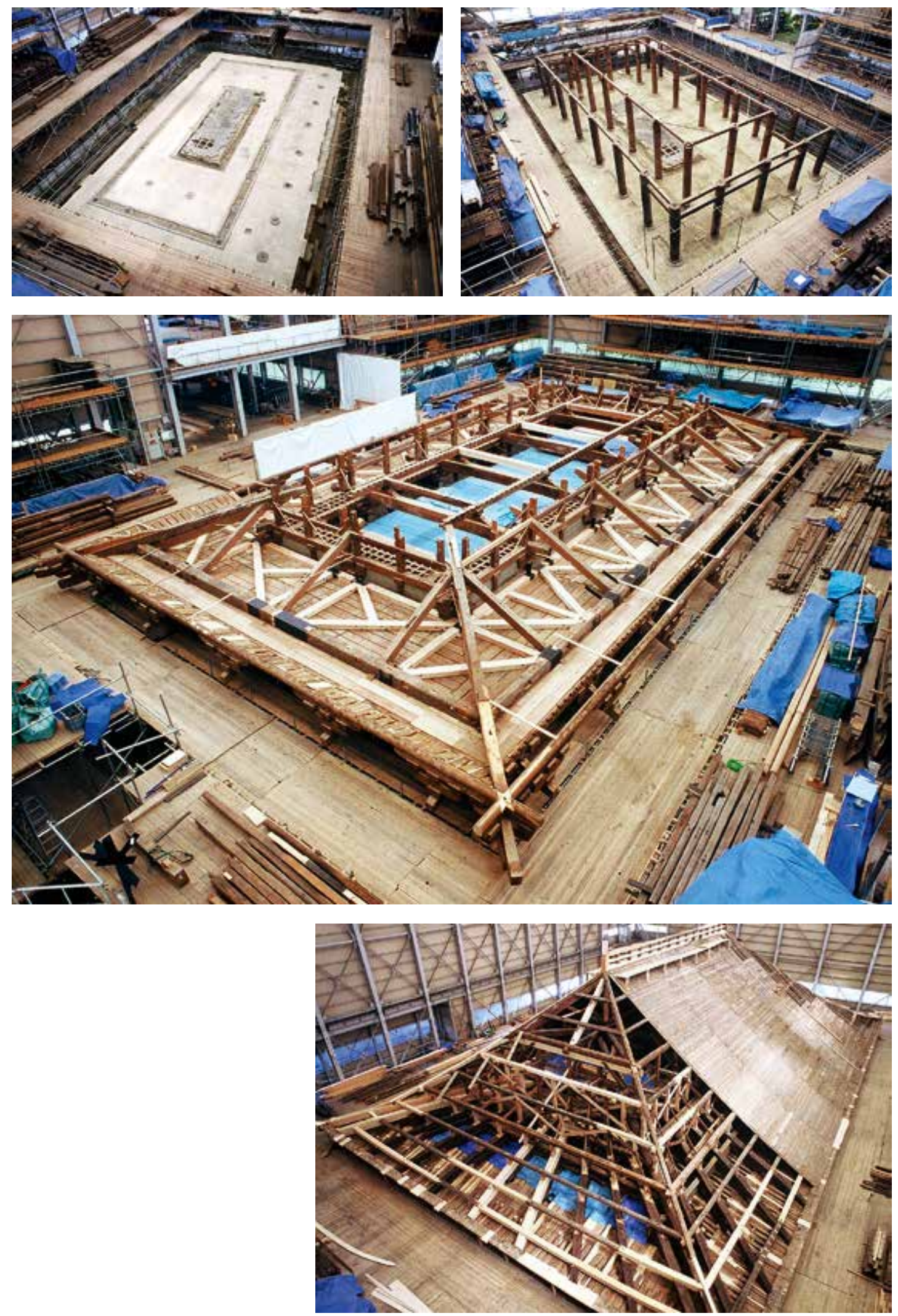
une ossature triangulée, à l'occidentale, a donc été insérée dans la partie haute de l'édifice. L'une des raisons de ce choix est en lien avec l'avenir. L'idée est qu'au cours du prochain démantèlement, d'ici cent, deux cents ou trois cents ans, les restaurateurs et les archéologues puissent identifier clairement les réparations qui ont été effectuées au tournant du deuxième millénaire de notre ère, sans qu'il y ait de confusions possibles avec les réparations effectuées par les différentes générations de restaurateurs qui ont précédé la nôtre. Une autre technique ou un autre matériau comme le métal aurait pu être choisi pour arriver au même résultat technique. Mais dans le cas du Tōshōdai-ji, pour marquer le changement de millénaire, les restaurateurs ont souhaité transmettre un travail réalisé avec des bois d'excellente qualité et envoyer un message sur l'évolution de leurs savoir-faire : ils respectent et s'astreignent à maîtriser les techniques ancestrales, mais ils accumulent toujours les savoir-faire venus d'ailleurs, dans ce cas ceux venus de l'Occident, et s'en nourrissent, tout comme l'ont fait leurs ancêtres avec les techniques chinoises par exemple.

Si vous le permettez, j'aimerais prendre un peu de recul par rapport à ce cas concret et rappeler que l'histoire de la restauration du patrimoine architectural au Japon couvre une période très large, du VII siècle à nos jours, et a touché aussi bien le pavillon principal du monastère bouddhique du Hōryū-ji, ou celui du Tōshōdai-ji sur lequel nous venons de nous attarder, mais aussi des édifices comme le Musée national d'art occidental conçu par Le Corbusier. Si les bâtiments concernés par les programmes de restauration sont des plus variés - des châteaux forts, des palais ou des maisons rurales, ou encore des temples bouddhiques et des sanctuaires shintō -, ces chantiers concernent dans leur majorité des édifices en bois, qui se détériorent rapidement - phénomène accéléré par les conditions climatiques et géologiques du pays : un degré d'humidité élevé, des typhons et des tremblements de terre. Ainsi les éléments les plus exposés sont-ils les toits, les avant-toits, les murs extérieurs et les parties enterrées. Sans entretien régulier, ces parties s'abîment encore plus vite, raison pour laquelle on effectue régulièrement des réparations. Ce contexte très particulier est, me semble-t-il, ce qui a permis à nos artisans de conserver et de transmettre leur savoir-faire au fil des générations. Aujourd'hui nous œuvrons pour que cette transmission se perpétue sans refuser les modernisations.

10a-d. Séquence photographique résumant le remontage complet du kondō (pavillon majeur cultuel) du monastère bouddhique Tōshōdai-ji,

à Nara, entre mars 2005 et août 2007. 


\section{Jean-Sébastien Cluzel}

Archéologue et architecte, Jean-Sébastien Cluzel enseigne l'histoire de l'art et l'archéologie de I'Extrême-Orient à la faculté des Lettres de Sorbonne Université. Spécialiste de I'histoire de l'architecture du Japon, il s'est occupé de la restauration des fabriques japonaises du jardin Albert-Kahn à Boulogne-Billancourt, de I'expertise de la salle des Cigognes pour le musée des Confluences à Lyon, et il est associé en tant qu'expert au projet de restauration du cinéma La Pagode à Paris. On retient parmi ses ouvrages : Architecture éternelle du Japon - De I'histoire aux mythes (Dijon, Faton, 2008) ; Hokusai - Le vieux fou d'architecture (Paris, Seuil / BnF, 2014) ; Le Japonisme architectural en France 1550-1930 (Dijon, Faton, 2018).

\section{Nishida Masatsugu}

Architecte et historien de l'architecture, Nishida Masatsugu est professeur au Kyōto Institute of Technology. Spécialiste d'architecture du Moyen Âge, il a dirigé de nombreuses campagnes de relevés sur l'architecture romane en France. Son ouvrage de synthèse, intitulé De la proportion de l'architecture cistercienne, paru en 2006 au Japon, a été récompensé par le prix de la Society of Architectural Historians of Japan. Spécialiste d'architecture comparée, il a également publié plusieurs ouvrages en français, dont le Vocabulaire de la spatialité japonaise (Paris, CNRS, 2014), Le sanctuaire d'Ise - Récit de la $62^{\mathrm{e}}$ reconstruction (Bruxelles, Mardaga, 2015).

\section{Yagasaki Zentarō}

Yagasaki Zentarō enseigne I'histoire de l'architecture et des jardins japonais (Osaka Electro-communication University). Spécialiste de l'architecture historique japonaise et plus spécialement de I'architecture résidentielle et de l'architecture du thé, il est très engagé dans les domaines de la conservation et de la restauration du patrimoine bâti. Continuellement associé à des chantiers de restauration de monuments classés patrimoine important, comme celui du grand temple bouddhique Higashi-Hongan-ji à Kyōto, il est aussi l'auteur d'articles, de rapports d'expertise architecturale et d'ouvrages en japonais sur I'histoire de I'architecture. II a notamment édité et publié l'édition critique d'un document classique portant sur la cérémonie du thé à l'époque pré-moderne : Chafu『茶譜』[Livre de thé], Tani Akira, Yagasaki Zentarō (éd.), 2 vol., Kyōto, Shibunkaku shuppan, 2010. II fut I'architecte-restaurateur de la villa et du jardin du style sukiya construit au début $\mathrm{XX}^{\mathrm{e}}$ siècle, Garyūsansō 臥龍山荘, dans la ville d'Ōzu.

\section{Yoshida Kōichi}

Yoshida Kōichi a longtemps enseigné I'histoire de l'architecture occidentale à l'Université nationale de Yokohama et est également connu pour ses travaux théoriques sur la conservation du patrimoine architectural. En tant que spécialiste de l'architecture française des $\mathrm{XIX}^{\mathrm{e}}$ et $\mathrm{XX}^{\mathrm{e}}$ siècles, il a conduit plusieurs enquêtes archéologiques sur l'architecture moderne à Kamakura et Yokohama. En 1997, sa recherche sur Auguste Perret et Tony Garnier a été récompensée par le prix de I'Architectural Institute of Japan (AIJ). Parmi ses derniers ouvrages, on notera Nihon no Seiki modanizumu kenchikuzō 『日本の盛期モダニズム 建築像』[Portraits de l'architecture moderniste à l'époque de son apogée au Japon] (Chiba-ken Matsudo-shi, Ōkokusha, 2019) et Nihon no Ārudeko no kenchiku monogatari 『日本のアール・デ コの建築物語』[L'architecture Art déco au Japon] (Chiba-ken Matsudo-shi, Ōkokusha, 2016).

\section{NOTES}

1. Françoise Choay, Laallégorie du patrimoine, Paris, Éditions du Seuil, 1992.

2. Alain Schnapp, La conquête du passé : aux origines de l'archéologie, Paris, Carré, 1993.

3. André Chastel et Jean-Pierre Babelon, La notion de patrimoine, Paris, éditions Liana Levi, 1994.

4. Pierre Nora (dir.), Les lieux de mémoire, Paris, Gallimard, 1994.

5. On se reportera à Knut Einar Larsen et Nils Marstein Conference on Authenticity in Relation to the World Heritage Convention, Preparatory Workshop, Bergen, Tapir Folag, 1994 ; Knut Einar Larsen (dir.), Conférence de Nara sur l'authenticité, Japon, compte-rendu novembre 1994, World Heritage Centre (Norvège), Unesco, 1995.

6. Certaines communications de ces différents colloques échappent bien entendu à ce constat. Sur le Japon, on peut noter la communication de Nicolas Fiévé, "Patrimoine et architecture au Japon : note sur les mots du monument historique ", dans Régis Debray (dir.), L'abus monumental, actes de colloque ("Entretiens du patrimoine », Paris, Théâtre national de Chaillot, 1998), Paris, Fayard - Éditions du Patrimoine, 1999, p. 323-345.

7. François Furet (dir.), Patrimoine, temps, espace : patrimoine en place, patrimoine déplacé, Paris, Fayard Éditions du Patrimoine, 1997.

8. Marcel Detienne, Comparer l'incomparable, Paris, Éditions du Seuil, 2000.

9. On se reportera à Nao Hayashi-Denis (dir.), Conférence internationale sur la sauvegarde du patrimoine culturel matériel et immatériel. Vers une approche intégrée, Unesco, Navis, 2006

10. On pourra se reporter à Nishida Masatsugu, JeanSébastien Cluzel, Philippe Bonnin, «Authenticité et 
restauration de la mémoire dans l'architecture monumentale japonaise ", dans Villes et Best practices, numéro thématique d'Espace et Société, no 131, 2007 / 4, p. 153170. Nicolas Fiévé et Yamasaki Masafumi, « La conservation des édifices classés au rang de bien culturel », et Nicolas Fiévé, "La protection des sites inscrits sur la Liste du Patrimoine mondial de l'UNESCO ", dans Nicolas Fiévé (dir.), Atlas historique de Kyōto, Paris, Éditions de I'UNESCO / Éditions de l'Amateur, 2008, p. 293-300 et p. 301-306. Christophe Marquet, "La notion de patrimoine archéologique et artistique à l'aube du Japon moderne ", dans Pierre-François Souyri et Jean-Paul Demoule (dir.), Archéologie et patrimoine au Japon, actes du colloque, Paris, Éditions de la Maison des Sciences de I'Homme, 2008, p. 107-118 ; Christophe Marquet, Arnaud Nanta, Laurent Nespoulous (dir.), Patrimonialisation et identités en Asie orientale, numéro thématique d'Ebisu. Études japonaises, n 52, 2014 [en ligne, DOI : https:// doi.org/10.4000/ebisu.1569].

11. Sur ce sujet, on pourra se reporter à Christoph Henrichsen (dir.), Historische Holzarchitektur in Japan: statische Ertüchtigung und Reparatur, Stuttgart, Theiss, 2003.

12. Sur ce sujet, on verra Jean-Sébastien Cluzel et Nishida Masatsugu (dir.), Le sanctuaire d'Ise - Récit de la $62^{\mathrm{e}}$ reconstruction, Bruxelles, Mardaga, 2015.

13. On trouvera les résultats de l'enquête archéologique conduite lors du démontage de ces pavillons, ainsi que la description de ce chantier dans Jean-Sébastien Cluzel et al., "Albert Kahn, archéologie d'un héritage nippon ", dans Jean-Sébastien Cluzel (dir.), Le japonisme architectural en France, 1550-1930, Dijon, Faton, 2018, p. 275-309.

14. Larsen, 1994-1995, cité n. 5 .

15. Disposition spirituelle et esthétique que l'on peut traduire par "tranquillité calme", NdIR.

16. On se reportera essentiellement à : Hiraga Amana 平賀 あまな, Koshajihozonhōjidai no kenzōbutsusūrishuhō to hozaongainen『古社寺保存法時代の建造物修理手法 と保存概念』 [La méthode de réparation de bâtiment et le concept de conservation à l'époque de la loi sur conservation des sanctuaires et des temples anciens], thèse de doctorat du Tōkyō Institute of Technology (Kōgyō Daigaku Hakase), 2001 ; Shimizu Shigeatsu 清水 重敦, Kenchiku hozon gainen no seiseishi 『建築保存 概念の生成史』 [Histoire de la genèse du concept de conservation architecturale], Tōkyō, Chuokoron Bijutsu Shuppan, 2013 ; Suzuki Masataka 鈴木正 崇編 (dir.), Ajia no bunka isan - kako, genzai, mirai 『アジアの文化遺産一過去·現在·未来』[Patrimoine culturel en Asie - passé, présent, futur], Tōkyō, Keiō Gijuku Daigaku Higashi Ajia Kenkyūjo [Keio Institute of East Asian Studies], 2015 ; Aoyagi Norimasa 青柳憲 昌, Nihon kindai no kenchiku hozon hōhōron: hōryūji shōwa daishūri to dōjidai no hozon rinen 『日本近代 の建築保存方法論:法隆寺昭和大修理と同時代の保存 理念』[Traité sur la méthode moderne de conservation architecturale à l'époque moderne au Japon], Tōkyō, Chuokoron Bijutsu Shuppan, 2019.

17. Eugène Viollet-le-Duc, Dictionnaire raisonné de l'architecture française du XI au XVI siècle, Paris, Librairiesimprimeries réunies, 1856.
18. Takayama Rinjirō 高山林次郎, «Koshaji oyobi kobijutsu no hozon o ronzu »「古社寺及び古美術の保存を 論ず」[Réflexion sur la conservation des sanctuaires et des temples anciens et des antiquités], dans Taiyō 『太陽』, n 10 , vol. 5, mai 1899, p. 51-62.

19. Tsuji Zennosuke 辻善之助, “Koshaji hozon no hōhō nitsuite no sehyou o ronzu »「古社寺保存の方法 に就ての世評を論ず」 [Réflexion sur la réputation publique pour la méthode de conservation des sanctuaires et temples anciens], dans Rekishichiri 『歴史 地理』, nº 2, vol. 3, février 1901, p. 91-100.

20. Basil Hall Chamberlain, Things Japanese, Being Notes on Various Subjects Connected with Japan, Londres, K. Paul, 1890.

21. Ce journal a été découvert pas deux historiens japonais, qui l'ont traduit et publié en japonais en 1933. On pourra se reporter à Gōdon Sumisu no Nippon gyōten nikki『ゴードン・スミスのニッポン仰天日記』 [Journal d'ébahissement de Gordon Smith au Japon], Aramata Hiroshi 荒俣宏 et Ōhashi Etsuko 大橋悦子訳 (trad. et éd.), Tōkyō, Shōgakukan, 1993, p. 240. On pourra également se reporter à Richard Gordon Smith, Travels in the Land of the Gods, 1898-1907: the Japan Diaries of Richard Gordon Smith, Victoria Manthorpe (éd.), New York, Prentice-Hall Press, 1986.

22. Lafcadio Hearn, Japan, an Attempt at Interpretation, New York, the Macmillan Company, 1904.

23. On se reportera à Luís Fróis, História do Japão (1583-1597), Lisbonne, Josef Wiki, 1976.

24. Alessandro Valignano, Les Jésuites au Japon : relation missionnaire (1583), J. Bésineau (trad. fra.), Paris, Desclée de Brouwer (coll. "Christus »), 1990.

25. On pourra se reporter aux Epistolæ S. Francisci Xaverii aliaque ejus scripta [Lettres de Saint François Xavier et ses autres écrits], Rome, Typ. Pontificiae universitatis Gregorianae (coll. "Monumenta Historica Societatis lesu », vol. 67 et 68), 1944-1945.

26. On pourra se reporter à François Caron, Le puissant royaume du Japon : la description de François Caron (1636), Jacques et Marianne Proust (trad. fra. et notes), Paris, Chandeigne, 2003.

27. Journal dont est tiré le livre, posthume : Engelbert Kæmpfer, The History of Japan, Giving An Account of the Ancient and Present State and Gouvernment of that Empire; of Its Temples, Palaces, Castles and Other Buildings; of Its Metals, Minerals, Trees, Plants, Animals, Birds and Fishes; of the Chronology and Succession of the Emperors, Ecclesiastical and Secular; of The Original Descent, Religions, Customs, and Manufactures of the Natives, and of Their Trade and Commerce with the Dutch and Chinese. Together with a Description of the Kingdom of Siam. Written in High-Dutch by Engelbertus Kaempfer, M. D. Physician to the Dutch Embassy to the Emperor's Court; and translated from his Original Manuscript, Johann Gaspar Scheuchzern (éd. et trad. angl.), Londres, 1727.

28. Philipp Franz von Siebold, Nippon. Archiv zur Beschreibung von Japan und dessen Neben- und Schutzländern jezo mit den südlichen Kurilen, Krafto, Koorai und denLiukiu-Inseln, nach japanischen undeuropäischen Schriften und eigenen Beobachtungen bearbeitet, 7 vol., Leyde, 1832-1852. 
29. Marco Polo, Le Devisement du monde, Le livre des merveilles, Arthur-Christopher Moule et Paul Peillot (éd.), Louis Hambis (trad. fra.), Paris, La Découverte, 2004.

30. Eugène Viollet-le-Duc, "Restauration », dans Dictionnaire raisonné de l'architecture française $d u X l^{\circ}$ au XVI siècle, 1854-1868, Paris, A. Morel, 1866, t. 8, p. 14.

31. Dans cet ouvrage, Viollet-le-Duc présente une maison dont il ne précise pas la localité, mais on comprend par le contexte donné qu'il s'agit d'une maison en Asie de l'Est. Il écrit : « II était rare que le fils d'un homme riche détruisît la maison de son père pour la reconstruire à neuf. Si elle tombait de vétusté, il se contentait d'élever, à côté des anciennes constructions, de nouveaux pavillons différant très-peu [sic], d'ailleurs, de ceux qu'il abandonnait à la ruine. » Citation tirée d'Eugène Viollet-le-Duc, Histoire de l'habitation humaine depuis les temps préhistoriques jusqu'à nos jours, Paris, Hetzel, 1875, p. 273.

32. Voir le texte en ligne : https://whc.unesco.org/fr/ conventiontexte/ (consulté le 30 mars 2020).

33. Voir la note 5, et le texte du « Document de Nara sur l'authenticité (1994) » : https://www.icomos.org/ fr/notre-reseau/comites-scientifiques-internationaux/ liste-des-comites-scientifiques-internationaux/179articles-en-francais/ressources/charters-andstandards/186-document-de-nara-sur-lauthenticite (consulté le 30 mars 2020).

34. Ambrogio Calepinum, Dictionarium latino lusitanicum, ac iaponicum ex Ambrosii Calepini volumine depromptum... In Amacusa in Collegio iaponico Societatis lesu cum facultate Superiorum, Amakusa (Japon), Colegio laponico Societatis lesu, 1595, 3 vol. réédités en fac-similé : Tōkyō, Tōyō bunko, 1950-1952.

35. Ce dictionnaire est établi à partir d'un dictionnaire français-hollandais édité par François Halma en 1781.

36. Ōta Hirotarō 太田博太郎, Nihon kenchiku-shi josetsu 『日本建築史序説』 [Introduction à l'histoire de l'architecture japonaise] (1946), Tōkyō, Shōkokusha, 1989. Sur cet auteur on se reportera à Particularités de I'architecture japonaise - Ōta Hirotarō, Jean-Sébastien Cluzel et Nishida Masatsugu (trad. fra., commentaires et notes), Paris, Nouvelles éditions Scala, 2020. 